\title{
Lista de cigarritas (Hemiptera: Cicadellidae) de Cusco, Perú
}

\section{Checklist of leafhoppers (Hemiptera: Cicadellidae) from Cusco, Peru}

\author{
Juan F. Costa ${ }^{1 *}$ y Pedro W. Lozada ${ }^{2}$
}

\begin{abstract}
* Autor para correspondencia:
1. Laboratorio de Entomología, Oficina C-333, Facultad de Ciencias Biológicas, Universidad Nacional de San Antonio Abad del Cusco (UNSAAC), Ciudad Universitaria de Perayoc, Av. De La Cultura $\mathrm{N}^{\circ}$ 733, Cusco, Perú.

Email Juan F. Costa:

jfrancosta@gmail.com

2. Investigador Asociado. Departamento de Entomología, Museo de Historia Natural (MUHN), Universidad Nacional Mayor de San Marcos (UNMSM). Lima, Perú.
\end{abstract}

Presentado: $\quad 13 / 09 / 2010$

\section{Introducción}

En el Perú, los estudios sobre los cicadélidos (cigarritas) se han desarrollado de manera puntual e incluyendo insectos plagas principalmente (Langlitz 1964, Ojeda et al. 1971, Santa María et al. 2001, Valdiviezo \& Villarreal 2002, Rasmussen et al. 2003). Los estudios de Lozada (1992a, 1992b, 1997) presentan listas de cicadélidos de Perú y de grupos específicos (Lozada \& León 1996, Lozada 2001).

En Cusco, pocos son los estudios realizados sobre Cicadellidae en áreas cultivadas o silvestres (Carrasco 1968, Venero-Gonzales 1991, Ormachea \& Vidal 1994, Yábar et al. 2002, Costa \& Lozada 2004, Lozada et al. 2009).

El listado que se presenta sigue el ordenamiento taxonómico de Dietrich y Rakitov (2002), Dietrich y Dmitriev (2003) y Dietrich $(2005,2006)$.

\section{Nota:}

Las localidades y/o especies marcadas con $\left(^{*}\right)$ son citadas en Lozada (1992b).

Las localidades y/o especies marcadas con $\left({ }^{* *}\right)$ son citadas en Lozada (1997).

\section{Subfamilia Cicadellinae}

TRIBU CICADELLINI

\section{Acrulogonia Young, 1977}

**A. incompta Young, 1977. Hacienda María, Cusco; Marcapata, Cusco.

\section{Amblyscarta Stål, 1869}

A. modesta Young, 1977. Pilcopata-Atalaya (790m), 11.iii.2002, J. F. Costa col., trampa de luz. PaucartamboPilcopata (950m), 12.iii.2002, J. F. Costa col, trampa de luz.

A. obscura Young, 1977. Pilcopata-Atalaya (790m), 11.iii.2002, J. F. Costa col., trampa de luz.

A. opulenta (Walker, 1851). Paucartambo-Pilcopata (950m), 12.iii.2002, J. F. Costa col., trampa de luz.

\section{Apulia Distant, 1908}

A. flora Distant, 1908. Paucartambo-Pilcopata (950m), 12.iii.2002, J. F. Costa col, trampa de luz.

A. hyala Distant, 1908. San Pedro, K'osñipata (Paucartambo, 1400m), 10.xii.2007, J. F. Costa \& J. C. Astete.

**A. resupinata Young, 1977. Santa Isabel, Cusco.

Begonalia Young, 1977

**B. hydra (Distant, 1908). Callanga.

\section{Borogonalia Young, 1977}

B. crinata Young, 1977. Saylla (Cusco), 30.viii.1973, C. Martínez col.; Saqsayhuamán (Cusco, 3600m), 30.iii.2002, J. F. Costa col., ex Baccharis polianta, ex poáceas; Tancarpata (Santiago, 3350m), 08.v.2002, J. F. Costa col., ex Baccharis sp; Pumamarca (San Sebastián, 3450m), 19.ix.2002, J. F. Costa col., ex varios; Tancarpata (Santiago, 3350m), 14.x.2002, J. F. Costa col., ex varios; San Sebastián, 14.xi.2002, M. Olivera col., ex Pyrus communis, Progreso (Wanchaq), 26.xi.2002, J. F. Costa col., ex Baccharis sp; Progreso (Wanchaq), 22.xi.2004, J. F. Costa col., ex Baccharis sp; Santa María (San Sebastián, 3450m), 08.iv.2006, M. Cardenas col., ex Escallonia resinosa, ex Colletia spinosissima.

B. impressifrons (Signoret, 1854). Saqsayhuamán (Cusco, 3600m), 30.iii.2002, J. F. Costa col., ex Baccharis polianta, ex poáceas; Tancarpata (Santiago, 3350m), 08.v.2002, J. F. Costa col., ex Baccharis sp; Pumamarca (San Sebastián, 3450m), 19.ix.2002, J. F. Costa col., ex varios; Tancarpata (Santiago, 3350m), 14.x.2002, J. F. Costa col., ex varios; San Sebastián, 14.xi.2002, M. Olivera col., ex Pyrus communis, Progreso (Wanchaq), 26.xi.2002, J. F. Costa col., ex Baccharis sp; Progreso (Wanchaq), 22.xi.2004, J. F. Costa col., ex Baccharis sp; Santa María (San Sebastián, 3450m), 08.iv.2006, M. Cardenas col., ex Escallonia resinosa, ex Colletia spinosissima.

\section{Caldwelliola Young, 1977}

${ }^{* *}$ C. selvola Young, 1977. Callanga. 


\section{Caragonalia Signoret, 1855}

C. carminata Signoret, 1855. Machupicchu (Urubamba), 08.v.1965, O. Ochoa col.

\section{Cephalogonalia Evans, 1947}

C. flabellula (Jacobi, 1905). Pichari, La Convención (1000m), 15.viii.2005, A. Bustamante col.

Cephalogonalia sp. Pichari, La Convención (1000m), 15.viii.2005, A. Bustamante col.

\section{Coronigoniella Young, 1977}

${ }^{* *}$ C. ostenta Young, 1977. Callanga.

\section{Cyclogonia Melichar, 1926}

C. scutellatula Osborn, 1851. Paucartambo-Pilcopata, K'osńipata (2400m), 08.iii.2002, J. F. Costa col, trampa de luz.

C. sumai Young, 1977. San Pedro, K'osńipata (Paucartambo, 1400m), 15.viii.2001, A. Bustamante col.; Pilcopata (Paucartambo, 565m), 23.ii.2002, J. F. Costa col., trampa de luz; Esperanza-Pillahuata, K'osñipata (Paucartambo, 2900m), 27.vii.2003, J. F. Costa col., ex varios.

${ }^{* *}$ C. praetextatula (Jacobi, 1905). Callanga.

\section{Diedrocephala Spinola, 1850}

${ }^{* *} D$. variegata (Fabricius, 1775). Callanga. Actualmente presenta una nueva combinación taxonómica: $D$. bimaculata (Gmelin, 1789), de acuerdo a McKamey (2007).

\section{Dilobopterus Signoret, 1850}

**D. fenestratus Young, 1977. Callanga; Marcapata.

D. jemima Distant, 1908. Sahuayaco, Quillabamba (La Convención, 800m), 20.iii.1996, R. Casafranca col; PaucartamboPilcopata (K'osńipata, 2400m), 09.iii.2003, J. F. Costa col; Miraflores, Lares (Calca, 1618m), 20.v.2003, A. Bustamante col, ex Solanum tuberosum.

D. obliquatulus Jacobi, 1905. Pilcopata (Paucartambo, 565m), 21.iv.1964, O. Ochoa col. Callanga**.

\section{Erythrogonia Melichar, 1926}

E. amicula Jacobi, 1905. Sahuayaco, Quillabamba (La Convención, 800m), 20.iii.1996, R. Casafranca col. Vilcanota**; Marcapata**

${ }^{* *}$ E. aurivagula (Jacobi, 1905). Marcapata.

${ }^{* *}$ E. eburata Melichar, 1926. Vilcanota.

${ }^{* *}$ E. imitatricula (Jacobi, 1905). Callanga; Santa Isabel, Cusco.

${ }^{* *}$ E. kokomona Medler, 1963. Marcapata.

${ }^{* *} E$. sociales Melichar, 1926. Marcapata.

E. triplicula Jacobi, 1905. Pilcopata (Paucartambo, 565m), 21.iv.1964, O. Ochoa col; Machupicchu (Urubamba, 2800m), 07.v.1965, C. Martínez col; Machupicchu (Urubamba, 2800m), 26.xi.1965, C. Martínez col; Machupicchu (Urubamba, 2800m), 17.ix.1983, E. Madera col; Paco Pacuni (Limbani, Puno, 898m), 15.xi.2006, J. F. Costa col. Marcapata**.

**E. warsula Medler, 1963. Marcapata.

\section{Hortensia Metcalf \& Bruner, 1936}

H. similis (Walker, 1851). Sahuayaco, Quillabamba (La Convención, 800m), 15.iii.1996, R. Casafranca col; Sahuayaco, Quillabamba (La Convención, 800m), 20.iii.1996, R. Casafranca col; Pilcopata, K'osñipata (Paucartambo, 565m), 08.xii.2001, Alfaro \& Bustamante col, ex Ananas comosus (piña); Pilcopata, K'osńipata (Paucartambo, 565m), 23.ii.2002, J. F. Costa col, trampa de luz.

\section{Iragua Melichar, 1926}

**I. montana Young, 1977. Vilcanota.

** I. perplexa Young, 1977. Marcapata.

\section{Jozima Young, 1977}

J. leucopa (Walker, 1858). según Young (1977). PilcopataAtalaya (Paucartambo, 790m), 11.iii.2002, J. F. Costa col, trampa de luz. Marcapata**.

\section{Juliaca Melichar, 1926}

**J. bilineata (Melichar, 1951). Callanga.

**J. simoni Young, 1977. Santa Isabel, Cusco; Callanga.

\section{Kogigonalia Young, 1977}

** K. cajana Young, 1977. Callanga.

\section{Lissoscarta Stål, 1869}

**L. nipata Young, 1977. Hacienda María, Cusco.

\section{Macugonalia Young, 1977}

M. cavifrons (Stål, 1862). Pilcopata-Atalaya (Paucartambo, 790m), 11.iii.2002, J. F. Costa col, trampa de luz.

M. leucomelas Walker, 1851. Pilcopata (Paucartambo, 565m), 23.ii.2002, J. F. Costa col, trampa de luz.

\section{Mesogonia Melichar, 1926}

M. callangana Young, 1977. Pilcopata-Atalaya (Paucartambo, 790m), 11.iii.2002, J. F. Costa col, trampa de luz. Callanga**.

M. olivatula Osborn, 1926. Pilcopata-Atalaya (Paucartambo, 790m), 11.iii.2002, J. F. Costa col, trampa de luz.

${ }^{* *}$ M. retrorsa Young, 1977. Callanga.

** M. suspecta Young, 1977. Paucartambo.

${ }^{* *}$ M. tolosa (Distant, 1908). Cusco.

Mucrometopia Melichar, 1925

${ }^{* *}$ M. caudata (Walker, 1851). Vilcanota.

\section{Onega Distant, 1908}

O. bracteata Young, 1977. Sahuayaco, Quillabamba (La Convención, 800m), 15.xi.1995, R. Casafranca col; Pilcopata (Paucartambo, 565m), 02.ii.2002, A. Bustamante; Callanga**.

Onega sp. San Pedro, K'osñipata (Paucartambo, 1400m), 15.viii.2001, A. Bustamante col; Paucartambo-Pilcopata (2400m), 08.iii.2002, J. F. Costa col, trampa de luz.

\section{Oragua Melichar, 1926}


${ }^{* *}$ O. nusinasa Young, 1977. Santa Isabel, Cusco; Marcapata.

O. partitula (Jacobi, 1905). Pilcopata (Paucartambo, 565m), 23.ii.2002, J. F. Costa col, trampa de luz.

\section{Pachitea Melichar, 1926}

**P. habernula (Jacobi, 1905). Callanga.

P. ryma Young, 1977. Paucartambo-Pilcopata (950m), 12.iii.2002, J. F. Costa col, trampa de luz; PaucartamboPilcopata (1130m), 13.iii.2002, J. F. Costa col, trampa de luz; Callanga**.

P. subflava Walker, 1851. Paucartambo-Pilcopata (950m), 12.iii.2002, J. F. Costa col, trampa de luz.

\section{Pamplona Melichar, 1926}

**P. spatulata Young, 1977. Callanga.

\section{Paromenia Melichar, 1926}

P. marginata Young, 1977. Paucartambo-Pilcopata (1130m), 10.iii.2002, J. F. Costa col, trampa de luz. Santa Isabel, Cusco** .

P. quimbayensis (Kulgatz \& Melichar, 1902). Trocha Unión, Acjanaco, K'osńipata (Paucartambo, 2750m), 31.xii.2001, J. F. Costa col, ex varios; Pilcopata (Paucartambo, 565m), 23.ii.2002, J. F. Costa col; Trocha Unión, Acjanaco, K'osñipata (Paucartambo, 2750m), 27.ii.2002, J. F. Costa col, ex varios; EsperanzaPillahuata, K'osñipata (Paucartambo, 2900m), 27.vii.2003, J. F. Costa col, ex varios.

\section{Pawiloma Young, 1977}

P. fulpae Young, 1977. Machupicchu (Urubamba, 2800m), 17.ix.1983, E. Madera col; Ucchuhuerta, Choquequirao (Anta, 1600m), 16.v.2002, Cáceres \& Huerta col. Machupicchu**.

P. sirochia Young, 1977. Trocha Unión, Acjanaco, K'osñipata (Paucartambo, 2750m), 31.xii.2001, J. F. Costa col, ex varios.

\section{Punahuana Young, 1977}

P. dyscrita Young, 1977. Trocha Unión, Acjanaco, K'osñipata (Paucartambo, 2750m), 31.xii.2001, J. F. Costa col, ex varios; Trocha Unión, Acjanaco, K'osñipata (Paucartambo, 2750m), 27.ii.2002, J. F. Costa col, ex varios. Callanga**; Marcapata**.

\section{Ramosulus Young, 1977}

R. corrugipennis (Osborn, 1926). Pilcopata (Paucartambo, 565m), 23.ii.2002, J. F. Costa col., trampa de luz.

** R. fulgidus (Melichar, 1932). Callanga.

R. phaedrus Young, 1977. Trocha Unión, Acjanaco, K'osñipata (Paucartambo, 2750m), 31.xii.2001, J. F. Costa col., ex varios; Trocha Unión, Acjanaco, K'osñipata (Paucartambo, 2750m), 27.ii.2002, J. F. Costa col., ex varios.

\section{Schildola Young, 1977}

**S. ductilis Young, 1977. Santa Isabel, Cusco.

\section{Sibovia China, 1927}

S. aprica Melichar, 1926. Miraflores, Lares (Calca, 1618m), 25.v.2003, A. Bustamante.
**S. picchitula Young, 1977. Machupicchu.

**S. praevia (Melichar, 1926). Cusco.

\section{Soosiulus Young, 1977}

**S. flanmidulus (Jacobi, 1905). Marcapata.

**S. seimunculus (Melichar, 1932). Marcapata.

\section{Stehlikiana Young, 1977}

S. halticula (Jacobi, 1905). Machupicchu (Urubamba, 2800m), 03.ii.1964, C. Martínez col; Machupicchu (Urubamba, 2800m), 26.xi.1965, C. Martínez col; Pilcopata (Paucartambo, 565m), 16.xi.1968, O. Ochoa col; Paucartambo-Pilcopata, K'osñipata (2400m), 08.iii.2002, J. F. Costa col, trampa de luz. Marcapata**.

**S. obscura (Jacobi, 1905). Marcapata.

**S. recurva (Jacobi, 1905). Callanga; Vilcanota.

Tipuana Melichar, 1926

** T. expallida Young, 1977. Hacienda María, Cusco.

Tortigonalia Young, 1977

**T. torta Young, 1977. Callanga.

T. treva Young, 1977. Pilcopata (565m), 23.ii.2002, J. F. Costa col, ex gramíneas. Callanga**

\section{Trichogonia Breddin, 1901}

T. costata (Signoret, 1853). Ttio (Wanchaq, 3330m), 23.vii.1963, O. Ochoa col, Huancaro (Santiago), 20.v.1973, C. Martínez col; Saqsayhuamán (Cusco, 3600m), 24.ii.1983, E. Madera col; Ticatica, 20.vii.1983, E. Madera col, Los Incas (Cusco), 02.xi.1995, J. Álvarez col; Pillao Matao (San Sebastián, 3350m), 13.iv.2002; Saqsayhuamán (Cusco, 3600m), 30.iii.2003, J. F. Costa col, ex Daucus carota, ex Brassica oleracea, ex B. campestris; Pampallaqta, Pisaq (Calca, 3340m), 25.iv.2002, Alfaro \& Bustamante col, ex Solanum tuberosum, ex Buddleia coriacea; Salineras (San Sebastián, 3380m), 14.iv.2002, J. F. Costa, ex asteráceas.

\section{Zaruma Melichar, 1926}

** $Z$. decolorata Young, 1977. Callanga.

TRIBU PROCONIINI

Abana Distant, 1908

${ }^{* *}$ A. horvathi Jacobi, 1905. Cusco.

\section{Acrogonia Stål, 1869}

**A. stylata Young, 1968. Hacienda María, Cusco; Callanga.

\section{Anacuerna Young, 1968}

A. centrolinea (Melichar, 1925). Tipón, 22.viii.1973, C. Martínez col; Saylla, 30.viii.1973, C. Martínez col; Perayoc (Cusco, 3370m), 22.ix.1973, C. Martínez col; Ticatica (Santiago), 20.vii.1983, E. Madera col; Pumamarca (San Sebastián, 3450m), 14.ix.2002, J. F. Costa col, ex Daucus carota; Pillao Matao (San Sebastián, 3350m), 25.vi.2004, J. F. Costa col, ex Daucus carota, ex Trifolium repens; Kayra (San Jerónimo, 
3210m), 14.ix.2004, K. Gonzáles col, ex Medicago sativa; Saylla, 22.X.2004, J. F. Costa col, ex grass.

\section{Deselvana Young, 1968}

D. longipennis (Melichar, 1925). Pilcopata (Paucartambo, 565m), 23.ii.2002, J. F. Costa col., trampa de luz.

\section{Dichrophleps Stål, 1869}

D. truncata (Young, 1968). Pilcopata-Atalaya (Paucartambo, 790m), 11.iii.2002, J. F. Costa col., trampa de luz. Hacienda María, Cusco**. Río K'osñipata**.

Diestostemma Amyot \& Serville, 1843

**D. blantoni Young, 1968. Callanga.

${ }^{* *} D$. dolosum (Melichar, 1924). Cusco.

**D. reticulatum (Melichar, 1924). Cusco.

Egidemia China, 1927

${ }^{* *}$ E. obtusata (Melichar, 1925). Marcapata.

\section{Homoscarta Melichar, 1926}

H. boliviana Schmidt, 1928. Pilcopata (Paucartambo, 565m), 23.ii.2002, J. F. Costa col., trampa de luz.

${ }^{* *} H$. superciliaris (Jacobi, 1905). Cusco.

\section{Ichthyobelus Melichar, 1925}

I. bellicosus Melichar, 1925. Sahuayaco (La Convención, 800m), 15.iii.1996, R. Casafranca col.

\section{Molomea Signoret, 1855}

**M. consorta (Melichar, 1925). Vilcanota.

M. guttulata Melichar, 1925. Sahuayaco (La Convención, 800m), 15.iii.1996, R. Casafranca col.

\section{Oncometopia Stål, 1869}

O. venata Schroeder, 1959. Shintuya, Salvación (Madre de Dios), 07.iii.1968, E. Gonzáles col.; Pilcopata (Paucartambo, 565m), 16.xi.1968, O. Ochoa col.

Oncometopia sp. Pilcopata (Paucartambo, $565 \mathrm{~m}$ ), 04.x.1963, O. Ochoa col.; Sahuayaco (La Convención, 800m), 18.iii.1996, R. Casafranca col.; Pilcopata (Paucartambo, 565m), 15.ii.2002, A. Bustamante col.; Pilcopata-Atalaya (Paucartambo, 790m), 11.iii.2002, J. F. Costa col., trampa de luz; Paucartambo-Pilcopata (950m), 12.iii.2002, J. F. Costa col., trampa de luz; Pilcopata (Paucartambo, 565m), 01.xi.2002, A. Bustamante col.

\section{Procandea Young, 1968}

**P. andina Young, 1968. Marcapata.

**P. cordillerae Young, 1968. Cusco.

P. inca Young, 1968. Pilcopata (Paucartambo, 565m), 15.xi.1967, O. Ochoa col.

**P. monticola Young, 1968. Santa Isabel, Cusco; Callanga.

** P. quechua Young, 1968. Callanga, Cusco.
Proconia Le Peletier \& Serville, 1825

P. marmorata Metcalf, 1965. Pilcopata (Paucartambo, 565m), 15.xi.1967, O. Ochoa col.

Proconopera Young, 1968

${ }^{* *}$ P. pullula (Jacobi, 1905). Callanga.

\section{Proconosama Young, 1968}

P. haenschi (Melichar, 1926). Sahuayaco (La Convención, 800m), 20.iii.1996, R. Casafranca col.; San Pedro, K'osñipata (Paucartambo, 1400m), 15.viii.2001, A. Bustamante col.

\section{Pseudometopia Schmidt, 1928}

P. irenae Young, 1968. San Pedro, K'osñipata (Paucartambo, Cusco, 1400m), 15.viii.2001, A. Bustamante col. Callanga**. Santa Isabel, Cusco**.

Rhaphirrhinus Laporte, 1832

$R$. phosphoreus (Linneus, 1758). Salvación (Madre de Dios), 17.xi.1968, O. Ochoa col.

\section{Subfamilia Deltocephalinae}

El concepto de esta Subfamilia fue recientemente ampliado para incluir a las Subfamilias Eupelicinae, Koebeliinae, Paraboloponinae, Penthiminae y Selenocephalinae; en Dietrich y Rakitov (2002) y Dietrich \& Dmitriev (2003).

\section{Tribu DeltocephalinI}

\section{Amplicephalus DeLong, 1926}

*A. auranticus Linnavuori \& DeLong, 1979. Machupicchu.

*A. paradoxus Linnavuori \& DeLong, 1976. Machupicchu.

Mattogrossus Linnavuori, 1959

${ }^{*}$ M. colonoides (Linnavuori, 1959). Quincemil, Cusco.

\section{Picchuia Linnavuori \& DeLong, 1979}

*P. pungens Linnavuori \& DeLong, 1979. Machupicchu.

\section{TRIBU DORATURINI}

\section{Icaia Linnavuori, 1973}

I. appendiculata Linnavuori \& DeLong, 1976. Huatata, Chinchero (Anta, 3800m), 15.viii.2004, J. F. Costa col., ex Solanum tuberosum; Huatata, Chinchero (Anta, 3800m), 25.vi.2005, J. F. Costa col., ex Solanum tuberosum, ex Vicia faba. Machupicchu*.

\section{TRIBU EUSCELINI}

\section{Andanus Linnavuori, 1959}

*A. bimaculatus Linnavuori, 1959. Quincemil, Cusco.

Atanus Oman, 1936

*A. picchuanus Linnavuori \& DeLong, 1976. Machupicchu.

\section{Bandaromimus Linnavuori, 1959}

B. fulvopictus Linnavuori, 1959. Pilcopata-Atalaya (Paucartambo, 790m), 11.iii.2002, J. F. Costa col., trampa de luz. 


\section{Brazosa Oman, 1936}

*B. picturella (Baker, 1923). Callanga (Paucartambo, Cusco).

\section{Chlorotettix Van Duzze, 1892}

Chlorotettix sp. Trocha Unión, Acjanaco, K'osñipata (Paucartambo, 2750m), 31.xii.2001, J. F. Costa col., ex varios; Trocha Unión, Acjanaco, K'osñipata (Paucartambo, 2750m), 27.ii.2002, J. F. Costa col., ex varios.

\section{Exitianus Ball, 1929}

${ }^{*}$ E. quadratulus (Osborn, 1923). Machupicchu.

\section{Paratanus Young, 1957}

P. exitiosus (Beamer, 1943). Pampallaqta, Lares (Calca, 3900m), 09.vii.2003, J. F. Costa col., ex gramíneas; Chilca, Ollantaytambo (Urubamba, 2600m), 12.v.2005, J. F. Costa col., ex Chenopodium quinoa, ex Zea mays; Cuncani, Lares (Calca, 3545m), 16.i.2004, J. F. Costa col, ex gramíneas; Tambohuaylla, Lares (Calca, 3510m), 17.i.2005, J. F. Costa col., ex Chenopodium quinoa; Marangani, Sicuani (Canchis), 22.v.2005, J. F. Costa col., ex Chenopodium quinoa, ex Zea mays.

P. yusti Young, 1957. Kayra (San Jerónimo, 3219m), 26.i.2002, J. F. Costa, ex Zea mays, ex Solanum tuberosum; San Jerónimo (San Jerónimo, 3400m), 13.iii.2002, J. F. Costa col., ex Zea mays, Phaseolus vulgaris; Saqsayhuamán (Cusco, 3600m), 28.vii.2002, J. F. Costa col., ex Baccharis polianta (chilca); Perayoc (Cusco, 3360m), 04.viii.2002, J. F. Costa col.; Pumamarca (San Sebastián, 3450m), 14.ix.2002, J. F. Costa col.; Tancarpata (San Sebastián, 3350m), 14.x.2002, J. F. Costa col., ex Zea mays; Amadeo Repeto (Santiago), 05.xi.2002, W. Cosio col.; Chilca, Ollantaytambo (Urubamba, 2600m), 12.v.2005, J. F. Costa col., ex Chenopodium quinoa, ex Zea mays; Pampallaqta, Lares (Calca, 3900m), 09.vii.2003, J. F. Costa col., ex gramíneas; Cuncani, Lares (Calca, 3545m), 16.i.2004, J. F. Costa col., ex gramíneas; Tambohuaylla, Lares (Calca, 3510m), 17.i.2005, J. F. Costa col., ex Chenopodium quinoa; Pisaq (Calca), 01.iv.2005, J. F. Costa col., ex Chenopodium quinoa; Marangani (Sicuani), 22.v.2005, J. F. Costa col., ex Chenopodium quinoa, ex Zea mays.

\section{Sinchonoa Linnavuori \& DeLong, 1978}

${ }^{*}$ S. machua DeLong, 1980. Machupicchu.

\section{Yungasia Linnavuori, 1959}

${ }^{*}$ Y. longipennis Linnavuori \& DeLong, 1976. Machupicchu. Tribu HECALINI

\section{Tenucephalus DeLong, 1944}

${ }^{*}$ T. saggitarius Linnavuori \& DeLong, 1976. Machupicchu.

\section{TRIBU MACROStelinI}

\section{Dalbulus DeLong, 1976}

D. maidis (DeLong \& Wolcott, 1923). Ollantaytambo (Urubamba, 2600m), 12.v.2005, J. F. Costa col., ex Chenopodium quinoa, ex Zea mays.

\section{Picchusteles Linnavuori \& DeLong, 1976}

${ }^{*}$ P. inca Linnavuori \& DeLong, 1976. Machupicchu.
TRIBU SCAPHYTOPIINI

\section{Scaphytopius Ball, 1931}

${ }^{*}$ S. atrifrons DeLong \& Linnavuori, 1978. Machupicchu.

TRIBU INCIERTA

\section{Amblysellus Sleesman, 1929}

Amblysellus sp. San Jerónimo (San Jerónimo, 3400m), 13.iv.2002, J. F. Costa col., ex Zea mays, ex Phaseolus vulgaris; Perayoc (Cusco, 3360m), 04.viii.2002, J. F. Costa col., ex Escallonia resinosa, ex gramíneas; Tancarpata (San Sebastián, 3350m), 14.x.2002, J. F. Costa col., ex varios; Amadeo Repeto (Santiago), 05.xi.2002, W. Cosio col., ex varios; Pampallaqta, Lares (Calca, 3900m), 09.vii.2003, J. F. Costa col., ex gramíneas; EsperanzaPillahuata, K'osñipata (Paucartambo, 2900m), 15.xi.2003, J. F. Costa col., ex gramíneas; Cuncani, Lares (Calca, 3545m), 16.i.2004, J. F. Costa col., ex gramíneas; Tambohuaylla, Lares (Calca, 3510m), 17.i.2005, J. F. Costa col., ex Chenopodium quinoa; Pumamarca (San Sebastián, 3350m), 16.vii.2005, J. F. Costa col.

\section{Bahita Osborn, 1923}

B. infuscata Osborn, 1923. Pilcopata (Paucartambo, 565m), 23.ii.2002, J. F. Costa col., ex gramíneas; Pilcopata-Atalaya (Paucartambo, 790m), 11.iii.2002, J. F. costa col., trampa de luz; Paucartambo-Pilcopata (Paucartambo, 1130m), 13.iii.2002, J. F. Costa col., trampa de luz.

B. irrorata Osborn, 1923. Paucartambo-Pilcopata (Paucartambo, 1130m), 13.iii.2002, J. F. Costa col., trampa de luz; Paucartambo-Pilcopata (Paucartambo, 950m), 12.iii.2002, J. F. Costa col., trampa de luz.

Bahita sp. Trocha Unión, Acjanaco, K'osñipata (Paucartambo, 2750m), 31.xii.2001, J. F. Costa col., ex Araceae, ex Conmelina sp (Conmelinaceae).

\section{Haldorus Oman, 1938}

Haldorus sp. Trocha Unión, Acjanaco, K'osñipata (Paucartambo, 2750m), 31.xii.2001, J. F. Costa col., ex varios; Trocha Unión, Acjanaco, K'osñipata (Paucartambo, 2750m), 27.ii.2002, J. F. Costa col., ex varios.

\section{Neomesus Linnavuori, 1959}

Neomesus sp. Saqsayhuamán (Cusco, 3600m), 28.vii.2002, J. F. Costa col., ex Baccharis polianta (chilca); Huatata, Chinchero (Anta, 3800m), 15.viii.2004, J. F. Costa col., ex Solanum tuberosum; Huatata, Chinchero (Anta, 3800m), 25.vi.2005, J. F. Costa col., ex Solanum tuberosum, ex Vicia faba.

\section{Subfamilia IAssinaE}

\section{TRIBU GYPONINI}

Anteriormente conocida como Subfamilia Gyponinae (= Scarinae, proveniente del género tipo Scaris Le Peletier \& Serville, 1825). Reducida a status de Tribu dentro de la Subfamilia IAssinae en Dietrich (2005).

\section{Acuera DeLong \& Freytag, 1972}

A. nana DeLong \& Freytag, 1974. Trocha Unión, Acja- 
naco, K'osñipata (Paucartambo, 2750m), 31.xii.2001, J. F. Costa col., ex varios; Pilcopata-Atalaya (Paucartambo, 790m), 11.iii.2002, J. F. Costa col., trampa de luz. Cusco, a lo largo del río K'osñipata (Cosñipata)**.

Acuera sp. Paucartambo-Pilcopata (Paucartambo, 1130m), 10.iii.2002, J. F. Costa col., ex varios; Pilcopata-Atalaya (Paucartambo, 790m), 11.iii.2002, J. F. Costa col., trampa de luz; Paucartambo-Pilcopata (Paucartambo, 950m), 12.iii.2002, J. F. Costa col., trampa de luz.

\section{Acuponana DeLong \& Freytag, 1970}

**A. enera DeLong \& Freytag, 1970. Hacienda María, Cusco.

\section{Barbatana Freytag, 1989}

**B. narda (DeLong \& Freytag, 1969). Hacienda Santa Isabel, Cusco.

\section{Chloronana DeLong \& Freytag, 1964}

${ }^{* *}$ C. decolora DeLong \& Freytag, 1964. Hacienda María, Cusco.

C. orbicula DeLong \& Freytag, 1964. Pilcopata-Atalaya (Paucartambo, 790m), 11.iii.2002, J. F. Costa col., trampa de luz.

${ }^{* *}$ C. rotunda DeLong \& Freytag, 1964. Callanga.

\section{Costanana DeLong \& Freytag, 1972}

Costanana sp. 1. Perayoc (Cusco, 3350m), 28.ix.1973, C. Martínez col.

Costanana sp. 2. Trocha Unión, Acjanaco, K'osñipata (Paucartambo, 2750m), 31.xii.2001, J. F. Costa col., ex varios; Trocha Unión, Acjanaco, K’osñipata (Paucartambo, 2750m), 27.ii.2002, J. F. Costa col., ex varios; Paucartambo-Pilcopata (Paucartambo, 2400m), 08.iii.2002, J. F. Costa col., trampa de luz; Paucartambo-Pilcopata (Paucartambo, 1130m), 13.iii.2002, J. F. Costa col., trampa de luz; Paucartambo-Pilcopata (Paucartambo, 950m), 12.iii.2002, J. F. Costa col., trampa de luz.

\section{Culumana DeLong \& Freytag, 1972}

${ }^{* *}$ C. bacula DeLong, 1979. Machupicchu.

${ }^{* *}$ C. concava DeLong, 1979. Santa Isabel, Cusco.

${ }^{* *}$ C. dualana DeLong, 1979. Santa Isabel, Cusco.

Curtara DeLong \& Freytag, 1976

${ }^{* *}$ C. picchua DeLong, 1979. Machupicchu.

${ }^{* *}$ C. sufflara DeLong, 1980. Callanga.

\section{Folicana DeLong \& Freytag, 1972}

** F. nota DeLong \& Freytag, 1972. Hacienda María, Cusco.

\section{Fuminana Freytag, 1989}

${ }^{* *}$ F. astra (DeLong \& Freytag, 1969). Callanga.

${ }^{* *}$ F. lira (DeLong \& Freytag, 1969). Hacienda María, Cusco.

\section{Gypona Germar, 1821}

${ }^{* *}$ G. adita DeLong \& Freytag, 1964. Santa Isabel, Cusco.

** G. fsura DeLong \& Freytag, 1964. Hacienda María, Cusco; Santa Isabel, Cusco.
G. glauca Fabricius, 1803. Trocha Unión, Acjanaco, K'osñipata (Paucartambo, 2750m), 31.xii.2001, J. F. Costa col., ex varios; Trocha Unión, Acjanaco, K'osñipata (Paucartambo, 2750m), 27.ii.2002, J. F. Costa col., ex varios.

** G. nacula DeLong \& Freytag, 1964. Santa Isabel, Cusco.

${ }^{* *} G$. nigrena DeLong \& Freytag, 1975. Callanga.

G. quadrina DeLong \& Linnavuori, 1977. Pilcopata (Paucartambo, 565m), 23.ii.2002, J. F. Costa col., ex asteráceas. Machupicchu**.

${ }^{* *} G$. solata DeLong \& Freytag, 1964. Callanga.

Gyponana Ball, 1920

G. apicata DeLong \& Freytag, 1964. Pilcopata (Paucartambo, 565m), 23.ii.2002, J. F. Costa col., ex asteráceas; PilcopataAtalaya (Paucartambo, 790m), 11.iii.2002, J. F. Costa col., trampa de luz.

\section{Hecalapona DeLong \& Freytag, 1975}

${ }^{* *}$ H. apicella DeLong, 1981. Callanga.

${ }^{* *} H$. berna DeLong \& Freytag, 1975. Callanga.

${ }^{* *}$ H. crinata DeLong \& Freytag, 1975. Callanga.

**H. eruva DeLong \& Freytag, 1975. Quincemil, Cusco.

${ }^{* *} H$. forceps DeLong \& Freytag, 1975. Callanga.

${ }^{* *}$ H. huella DeLong \& Freytag, 1975. Callanga.

${ }^{* *}$ H. scella DeLong \& Freytag, 1975. Callanga.

\section{Nulana DeLong, 1976}

${ }^{* *} N$. sinchona DeLong \& Martinson, 1980. Santa Isabel, Cusco.

${ }^{* *} N$. verdana DeLong \& Martinson, 1980. Hacienda María, Cusco.

\section{Polana DeLong, 1942}

**P. bulba DeLong \& Freytag, 1972. Callanga.

**P. concinna (Stål, 1862). Callanga.

**P. falsa DeLong \& Freytag, 1972. Hacienda María, Cusco.

**P. fetera DeLong \& Freytag, 1972. Hacienda María, Cusco; Santa Isabel, Cusco.

P. icara DeLong \& Freytag, 1972. Paucartambo-Pilcopata (Paucartambo, 1130m), 13.iii.2002, J. F. Costa col., trampa de luz. Santa Isabel, Cusco**.

**P. inimicus DeLong, 1976. Callanga.

**P. Lanara DeLong \& Freytag, 1972. Hacienda María, Cusco; Santa Isabel, Cusco.

**P. resupina DeLong \& Freytag, 1972. Hacienda María, Cusco.

**P. scruta DeLong \& Freytag, 1972. Hacienda María, Cusco.

Polana sp. Paucartambo-Pilcopata (Paucartambo, 1130m), 13.iii.2002, J. F. Costa col., trampa de luz; PaucartamboPilcopata (Paucartambo, 950m), 12.iii.2002, J. F. Costa col., 
trampa de luz; Esperanza-Pillahuata, K'osñipata (Paucartambo, $2821 \mathrm{~m})$, 27.vii.2003, J. F. Costa col., ex gramíneas.

\section{Ponana DeLong \& Freytag, 1967}

${ }^{* *}$ P. atea DeLong \& Freytag, 1967. Santa Isabel, Cusco.

${ }^{* *}$ P. avena DeLong \& Freytag, 1967. Santa Isabel, Cusco.

** P. berta DeLong \& Freytag, 1967. Santa Isabel, Cusco.

**P. cerosa DeLong \& Freytag, 1967. Pillahuata (Paucartambo).

** P. hilara DeLong \& Martinson, 1973. Santa Isabel, Cusco.

**P. perusana DeLong, 1980. Santa Isabel, Cusco.

Ponana sp. Trocha Unión, Acjanaco, K'osñipata (Paucartambo, 2750m), 31.xii.2001, J. F. Costa col., ex varios; Paucartambo-Pilcopata (Paucartambo, 2400m), 09.iii.2003, J. F. Costa col., trampa de luz; Paucartambo-Pilcopata (Paucartambo, 1130m), 13.iii.2002, J. F. Costa col., trampa de luz; Paucartambo-Pilcopata (Paucartambo, 950m), 12.iii.2002, J. F. Costa col., trampa de luz.

\section{Ponanella DeLong \& Freytag, 1969}

** P. rubravenosa DeLong \& Freytag, 1969. Callanga.

\section{Scaris Le Peletier \& Serville, 1825}

**S. amputa (DeLong \& Freytag, 1969). Hacienda María, Cusco.

**S. caballa (DeLong \& Freytag, 1969). Hacienda María, Cusco.

**S. defecta (DeLong \& Freytag, 1969). Río Urubamba, Cusco. Cusco.

** fimbriella (DeLong \& Freytag, 1969). Hacienda María,

**S. maculosa (DeLong \& Freytag, 1969). Santa Isabel, Cusco.

S. serosa DeLong \& Freytag, 1967. Pilcopata-Atalaya (Paucartambo, 790m), 11.iii.2002, J. F. Costa col., trampa de luz.

Scaris sp. Santa Cruz, Salvación (Manu, Madre de Dios, 300m), 10.viii.2003, A. Bustamante col.

\section{Tenuacia DeLong, 1977}

** T. rubera DeLong, 1977. Santa Isabel, Cusco; Hacienda María, Cusco.

\section{Tuberana DeLong \& Freytag, 1971}

T. tubera DeLong \& Freytag, 1971. Pilcopata (Paucartambo, 565m), 11.x.1968, I. Ceballos col.

TRIBU INCIERTA

Grunchia Kramer, 1963

${ }^{* *}$ G. grossa (Linnavuori, 1957). Callanga.

\section{SubFAMILIA LEDRINAE}

Tribu XerophloeinI

\section{Xerophloea Germar, 1839}

$X$ viridis (Fabricius, 1794) [Cercopis]. Limatambo (Anta), 23.i.1982, E. Madera col.

\section{Subfamilia Megophthalminae}

\section{Tribu AgallinI}

Anteriormente conocida como Subfamilia Agallinae, recientemente reducida a categoría de Tribu dentro de la Subfamilia Megophthalminae (Dietrich, 2005).

\section{Agallia Curtis, 1883}

Agallia sp. Sahuayaco (La Convención, 800m), 20.iii.1996, R. Casafranca col.; Paucartambo-Pilcopata (Paucartambo, 1130m), 13.iii.2002, J. F. Costa col., trampa de luz. Trocha Unión, Acjanaco, K'osñipata (Paucartambo, 2750m), 31.xii.2001, J. F. Costa col., ex varios; Pilcopata-Atalaya (Paucartambo, 790m), 11.iii.2002, J. F. Costa col., trampa de luz; Esperanza-Pillahuata, K'osñipata (Paucartambo, 2900m), 27.vii.2003, J. F. Costa col., ex varios;

\section{Agalliopsis Kirkaldy, 1907}

${ }^{*} A$. atahualpa Linnavouri \& DeLong, 1979. Machupicchu.

*A. bicuspidata Linnavouri \& DeLong, 1979. Machupicchu.

A. cervina Oman, 1933. Paucartambo-Pilcopata (Paucartambo, 950m), 12.iii.2002, J. F. Costa col., trampa de luz.

*A. peruviana Oman, 1933. Callanga.

${ }^{*}$ A. spinosa Linnavouri \& DeLong, 1979. Machupicchu.

*A. vittata Linnavouri \& DeLong, 1979. Machupicchu.

\section{Subfamilia TypHLOCYBINAE}

\section{TRIBU EMPOASCINI}

\section{Empoasca Walsh, 1862}

E. kraemeri Ross \& Moore, 1957. Cusco. Citada por Carrasco (1968).

Empoasca sp. 1. San Jerónimo (San Jerónimo, 3400m), 13.iv.2002, J. F. Costa col., ex Zea mays, ex Phaseolus vulgaris; Perayoc (Cusco, 3360m), 04.viii.2002, J. F. Costa col., ex Escallonia resinosa, ex gramíneas; Tancarpata (San Sebastián, 3350m), 14.x.2002, J. F. Costa col., ex varios; Amadeo Repeto (Santiago), 05.xi.2002, W. Cosio col., ex varios; Pampallaqta, Lares (Calca, 3900m), 09.vii.2003, J. F. Costa col., ex gramíneas; EsperanzaPillahuata, K'osñipata (Paucartambo, 2900m), 15.xi.2003, J. F. Costa col., ex gramíneas; Cuncani, Lares (Calca, 3545m), 16.i.2004, J. F. Costa col., ex gramíneas; Tambohuaylla, Lares (Calca, 3510m), 17.i.2005, J. F. Costa col., ex Chenopodium quinoa; Pumamarca (San Sebastián, 3350m), 16.vii.2005, J. F. Costa col.

Empoasca sp. 2. San Jerónimo (San Jerónimo, 3400m), 13.iv.2002, J. F. Costa col., ex Zea mays, ex Phaseolus vulgaris; Perayoc (Cusco, 3360m), 04.viii.2002, J. F. Costa col., ex Escallonia resinosa, ex gramíneas; Tancarpata (San Sebastián, 3350m), 14.x.2002, J. F. Costa col., ex varios; Amadeo Repeto (Santiago), 05.xi.2002, W. Cosio col., ex varios; Pampallaqta, Lares (Calca, 3900m), 09.vii.2003, J. F. Costa col., ex gramíneas; Esperanza-Pillahuata, K'osñipata (Paucartambo, 2900m), 15.xi.2003, J. F. Costa col., ex gramíneas; Cuncani, Lares (Calca, 3545m), 16.i.2004, J. F. Costa col., ex gramíneas; Tambohuaylla, Lares (Calca, 3510m), 17.i.2005, J. F. Costa col., ex Chenopodium quinoa; Pumamarca (San Sebastián, 3350m), 16.vii.2005, J. F. Costa col. 


\section{Subfamilia Xestocephalinae}

\section{TRIBU PORTANINI}

\section{Portanus Ball, 1932}

\section{*P. boliviensis (Baker, 1923). Vilcanota.}

Portanus sp. Saqsayhuamán (Cusco, 3600m), 30.iii.2002, J. F. Costa col., ex Baccharis polianta, ex gramíneas, ex asteráceas.

\section{Discusión}

Las especies de cigarritas descritas en el mundo sobrepasan las 23 mil (Dietrich 2006) aunque las publicaciones con descripciones de nuevas especies está en aumento (por ejemplo: Argentina (Szwedo 2002); Brasil (Zahniser \& Webb 2004, Takiya \& Cavichioli 2004, Carvalho \& Cavichioli 2005); Colombia (Takiya \& Cavichioli 2004, Vargas 2006), Perú (Lozada 1992c, 1992d, 2001; Lozada \& León 1996, Zahniser \& Webb, 2004)).

En la región Neotropical se tienen registradas más de 4839 especies (Freytag \& Sharkey 2002). En Perú, dos listados de especies de cicadélidos dieron 634 especies contenidas en 114 géneros (Lozada 1992b, 1997) lo que representaría aproximadamente $13 \%$ de las especies registradas en el Neotrópico aunque este porcentaje ha aumentado debido a los nuevos registros de las especies y descripciones de especies nuevas de cicadélidos y especies que no fueron citadas por Lozada (1992b, 1997); por ejemplo: 17 especies de Empoasca de la Subfamilia Typhlocybinae (Langlitz 1964) y especies asociadas a plantas cultivadas y silvestres (Ojeda et al. 1971), 3 especies nuevas de la Subfamilia Cicadellinae: Ladoffa aguilari, Tortigonalia longicaudata, Cephalogonalia blancasi (Lozada 1992c, 1992d; Lozada \& León 1996), 8 especies de Amblysellus de la Subfamilia Deltocephalinae (Lozada 2001). Teniendo en cuenta este hecho, para Colombia se reportaron 679 especies (Freytag \& Sharkey 2002), mayor al número de especies reportadas para Perú. A pesar de esto, Perú puede contener un mayor número de especies ya que no se han realizado estudios completos sobre cicadélidos.

De las 634 especies reportadas para Perú por Lozada (1992b, 1997), 72 géneros y 136 especies fueron citadas para diferentes localidades de Cusco. El número de especies de Cusco represen$\tan 21,5 \%$ de las especies registradas para Perú. Actualmente, con el presente reporte se incrementa a 111 géneros y 203 especies registradas para Cusco.

\section{Agradecimientos}

Al Dr. Erick Yábar por su apoyo en el Laboratorio de Entomología. Idea Wild contribuyó con equipo de campo y la Asociación Biodiversidad y Desarrollo (B \& D) proveyó apoyo logístico. A aquellos investigadores que se dedicaron a la colecta y estudio de cicadélidos en la región Cusco.

\section{Literatura citada}

Carrasco Z., F. 1968. Lista Preliminar de Insectos del Departamento de Entomología. Rev. Fac. Ciencias, 2: 177-191. Cusco.

Carvalho A. N. \& R. R. Cavichioli. 2005. Portanus aliceae sp. nov. do Brasil (Hemiptera: Cicadellidae, Xestocephalinae). Neotropical Entomology, 34(2): 251-254.

Costa J. F. \& P. W. Lozada. 2004. Cigarritas (Homoptera: Cicadellidae) en el Valle del Cusco. XLVI Convención Nacional de Entomología, $N^{\circ} 08$. Arequipa.

Dietrich C. H. 2005. Keys to the families of Cicadomorpha and subfamilies and tribes of Cicadellidae (Hemiptera: Auchenorrhyncha). Florida Entomol., 88: 502-517.
Dietrich C.H. 2006. Guide to Subfamilies of Leafhoppers (Cicadellidae). Center for Biodiversity. Illinois Natural History Survey-INHS. Illinois. Pp. 44.

Dietrich C.H. \& D. A. Dmitriev. 2003. Reassessment of the leafhopper tribes Koebeliini and Grypotini Haupt (Hemiptera: Cicadellidae). Ann. Entomol. Soc. America, 96 (6): 766-775.

Dietrich C.H. \& R.A. Rakitov. 2002. Some remarkable new Deltocephalinae leafhoppers (Hemiptera: Cicadellidae: Deltocephalinae) from the amazonian rainforest canopy. J. New York Entomol. Soc. 110: 1-48.

Freytag P.H. and M.J. Sharkey. 2002. A preliminary list of the leafhoppers (Homoptera: Cicadellidae) of Colombia. Biota Colombiana, 3 (2): 235-283.

Langlitz E.R. 1964. The Economic Species of Empoasca in the Coastal and Sierra Regions of Perú. Rev. Per. Ent. 7: 54-70.

Lozada P.W. 1992a. Notas sobre Cicadellidae (Homoptera) en plantas forrajeras de Loreto, Perú. Rev. Per. Ent. 35: 24-26.

Lozada P.W. 1992b. Cicadellidae (Homoptera) registrados para el Perú. I: Xestocephalinae, Agalliinae y Deltocephalinae. Rev. Per. Ent. 35: 27-30.

Lozada P.W. 1992c. Una nueva especie de Ladoffa Young, 1977 (Homoptera: Cicadellidae). Rev. Per. Ent. 34: 61-62.

Lozada P.W. 1992d. Una nueva especie de Tortigonalia Young, 1977 (Homoptera: Cicadellidae). Rev. Per. Ent. 34: 63-64.

Lozada P.W. 1997. Cicadellidae (Homoptera) registrados para el Perú. II: Iassinae, Gyponinae y Cicadellinae. Rev. Per. Ent. 40: 27-36.

Lozada P.W. 2001. Las especies sudamericanas del Género Amblysellus SLEESMAN (Homoptera: Cicadellidae). En XLIII Convención Nacional de Entomología, $\mathrm{N}^{\mathrm{o}} 14$. Huancayo.

Lozada P.W., J. F. Costa \& E. Yábar. 2009. Estudio Preliminar de las "Cigarritas" (Hemiptera: Cicadellidae) del Cusco, Perú. L Convención Nacional de Entomología. Sociedad Entomológica del Perú (SEP) y Universidad Nacional Jorge Basadre Grohman (UNJBG). 08-12 Feb. Tacna, Perú.

Lozada P.W. \& C. León A. 1996. El Género Cephalogonalia Evans, 1947 (Homoptera: Cicadellidae) en el Perú. Rev. Per. Ent. 39: $11-12$.

McKamey S.H. 2007. Taxonomic catalogue of the leafhoppers (Membracoidea). Part 1. Cicadellinae. Memoirs of the American entomológical Institute 78: 394 pp.

Ojeda P., C. Ruíz \& M. Castro. 1971. Insectos del departamento de La Libertad y sus plantas hospederas cultivadas y silvestres. Rev. Mus. Hist. Nat., Departamento de Entomología. Universidad Nacional de Trujillo. Trujillo.

Ormachea E. \& D. Vidal. 1994. Evaluación de Dalbulus maidis como principal vector del puca-poncho en cultivos de maíz en el Valle Sagrado de los Incas. Universidad Nacional de San Antonio Abad del Cusco. Cusco.

Rasmussen C., A. Lagnaoui \& P. Esbjerg. 2003. Advances in the Knowledge of Quinoa Pests. Foods Review International 19 (1 \& 2): 61-75.

Santa María K., K. Zúñiga \& E. Núñez. 2001. Control etológico de Empoasca sp. (Homoptera: Cicadellidae) e identificación de cicadélidos en cultivos de fríjol (Phaseolus vulgaris). XLIII Convención Nacional de Entomología, N 78 . Huancayo.

Szwedo J. 2002. Studies on Xerophloeini leafhoppers with description of Pariacaca icanoensis gen. and sp. nov. from Argentina (Hemiptera: Cicadomorpha: Cicadellidae: Ledrinae). Genus 13 (2): 153-163.

Takiya D. M. \& R. R. Cavichioli. 2004. A review of the Neotropical sharpshooter genus Onega Distant, 1908 (Hemiptera: Cicadellidae: Cicadellini). Zootaxa, 718: 1-19.

Valdiviezo L., G. \& J. A. Villarreal. 2002. Insectos que infestan las principales plantas ornamentales en la ciudad de Piura. XLIV Convención Nacional de Entomología, Nº 59. Piura. 
Vargas R.J.M. 2004. Reconocimiento taxonómico del género Soosiulus (Hemiptera, Auchenorrhyncha, Cicadellidae) en Colombia. Universidad Nacional de Colombia. 10 pág.

Venero-Gonzales J. (1991) Trichogonia costata (Homoptera: Cicadellidae) en Buddleia coriacea (Loganiaceae). Rev. Per. Ent. 34: 65-67.

Yábar E., E. Echegaray \& E. Gianoli. 2002. Insect pests and natural enemies in two varieties of quinoa (Chenopodium quinoa) at Cusco, Peru. J. Appl. Ent., 126: 275-280.
Young D.A. 1977. Taxonomic study of the Cicadellinae, Part II. North Carol. Agric. Exp. Stat. Tech. Bull., 239: 1-1135.

Zahniser J. \& M.D. Webb. 2004. Placement of the Faltala Oman Leafhopper Group (Hemiptera: Cicadellidae: Deltocephalinae) with Descriptions of Three New Species. Ann. Entomol. Soc. Am., 97(4): 667-674.

Apéndice 1. Lista de especies de Cicadellidae y localidades reportadas incluyendo coordenadas y altitud. Las especies marcadas con asterisco fueron citadas en Lozada (1992b, 1997).

\begin{tabular}{|c|c|c|c|c|c|c|}
\hline Subfamilia & Tribu & Especie & Longitud & Latitud & Altitud & Localidad \\
\hline Cicadellinae & Cicadellini & Acrulogonia incompta* & -71.390 & -12.910 & 800 & Hacienda María* \\
\hline Cicadellinae & Cicadellini & Acrulogonia incompta* & -70.894 & -13.503 & 1880 & Marcapata* \\
\hline Cicadellinae & Cicadellini & Amblyscarta opulenta & -71.430 & -13.170 & 950 & Paucart-Pilcopata \\
\hline Cicadellinae & Cicadellini & Amblyscarta modesta & -71.300 & -12.940 & 790 & Atalaya \\
\hline Cicadellinae & Cicadellini & Amblyscarta modesta & -71.430 & -13.170 & 950 & Paucart-Pilcopata \\
\hline Cicadellinae & Cicadellini & Amblyscarta obscura & -71.300 & -12.940 & 790 & Atalaya \\
\hline Cicadellinae & Cicadellini & Apulia resupinata* & -71.550 & -13.060 & 1590 & Santa Isabel* \\
\hline Cicadellinae & Cicadellini & Apulia hyala & -71.560 & -13.100 & 1400 & San Pedro \\
\hline Cicadellinae & Cicadellini & Apulia flora & -71.430 & -13.170 & 950 & Paucart-Pilcopata \\
\hline Cicadellinae & Cicadellini & Begonalia hydra* & -71.540 & -12.820 & 1500 & Callanga* \\
\hline Cicadellinae & Cicadellini & Borogonalia crinata & -71.980 & -13.500 & 3600 & Saqsayhuaman \\
\hline Cicadellinae & Cicadellini & Borogonalia crinata & -71.940 & -13.540 & 3350 & Tancarpata \\
\hline Cicadellinae & Cicadellini & Borogonalia crinata & -71.920 & -13.510 & 3580 & Pumamarca \\
\hline Cicadellinae & Cicadellini & Borogonalia crinata & -71.930 & -13.530 & 3290 & San Sebastián \\
\hline Cicadellinae & Cicadellini & Borogonalia crinata & -71.960 & -13.520 & 3320 & Wanchaq \\
\hline Cicadellinae & Cicadellini & Borogonalia crinata & -71.890 & -13.510 & 3450 & Santa María \\
\hline Cicadellinae & Cicadellini & Borogonalia crinata & -71.820 & -13.560 & 3150 & Saylla \\
\hline Cicadellinae & Cicadellini & Borogonalia impressifrons & -71.980 & -13.500 & 3600 & Saqsayhuaman \\
\hline Cicadellinae & Cicadellini & Borogonalia impressifrons & -71.940 & -13.540 & 3350 & Tancarpata \\
\hline Cicadellinae & Cicadellini & Borogonalia impressifrons & -71.920 & -13.510 & 3580 & Pumamarca \\
\hline Cicadellinae & Cicadellini & Borogonalia impressifrons & -71.930 & -13.530 & 3290 & San Sebastián \\
\hline Cicadellinae & Cicadellini & Borogonalia impressifrons & -71.960 & -13.520 & 3320 & Wanchaq \\
\hline Cicadellinae & Cicadellini & Borogonalia impressifrons & -71.890 & -13.510 & 3450 & Santa Maria \\
\hline Cicadellinae & Cicadellini & Caragonalia carminata & -72.550 & -13.170 & 2450 & Machupicchu* \\
\hline Cicadellinae & Cicadellini & Cephalogonalia flabellula* & -72.703 & -12.836 & 1060 & Quillabamba* \\
\hline Cicadellinae & Cicadellini & Cephalogonalia flabellula & -73.830 & -12.510 & 1000 & Pichari \\
\hline Cicadellinae & Cicadellini & Cephalogonalia sp & -73.830 & -12.510 & 1000 & Pichari \\
\hline Cicadellinae & Cicadellini & Coronigoniella ostenta* & -71.540 & -12.820 & 1500 & Callanga* \\
\hline Cicadellinae & Cicadellini & Cyclogonia praetextatula* & -71.540 & -12.820 & 1500 & Callanga* \\
\hline Cicadellinae & Cicadellini & Cyclogonia scutellatula & -71.590 & -13.140 & 2400 & Paucart-Pilcopata \\
\hline Cicadellinae & Cicadellini & Cyclogonia sumai & -71.560 & -13.100 & 1400 & San Pedro \\
\hline Cicadellinae & Cicadellini & Cyclogonia sumai & -71.430 & -12.980 & 565 & Pilcopata \\
\hline Cicadellinae & Cicadellini & Cyclogonia sumai & -71.580 & -13.150 & 2900 & Esperanza \\
\hline Cicadellinae & Cicadellini & Cyclogonia sumai & -71.790 & -13.120 & 2628 & Pillahuata \\
\hline Cicadellinae & Cicadellini & Diedrocephala variegata* & -71.540 & -12.820 & 1500 & Callanga* \\
\hline Cicadellinae & Cicadellini & Dilobopterus jemima & -72.510 & -12.680 & 800 & Sahuayaco \\
\hline Cicadellinae & Cicadellini & Dilobopterus jemima & -71.590 & -13.140 & 2400 & Paucart-Pilcopata \\
\hline Cicadellinae & Cicadellini & Dilobopterus jemima & -72.150 & -12.810 & 1618 & Miraflores \\
\hline Cicadellinae & Cicadellini & Dilobopterus jemima & -71.430 & -12.980 & 565 & Pilcopata \\
\hline Cicadellinae & Cicadellini & Dilobopterus jemima* & -70.894 & -13.503 & 1880 & Marcapata* \\
\hline Cicadellinae & Cicadellini & Dilobopterus jemima* & -71.540 & -12.820 & 1500 & Callanga* \\
\hline Cicadellinae & Cicadellini & Dilobopterus obliquatulus* & -71.540 & -12.820 & 1500 & Callanga* \\
\hline Cicadellinae & Cicadellini & Dilobopterus obliquatulus & -71.430 & -12.980 & 565 & Pilcopata \\
\hline Cicadellinae & Cicadellini & Erythrogonia amicula* & -71.033 & -14.483 & 4240 & Vilcanota* \\
\hline Cicadellinae & Cicadellini & Erythrogonia amicula* & -70.894 & -13.503 & 1880 & Marcapata* \\
\hline Cicadellinae & Cicadellini & Erythrogonia amicula & -72.510 & -12.680 & 800 & Sahuayaco \\
\hline Cicadellinae & Cicadellini & Erythrogonia aurivagula* & -70.894 & -13.503 & 1880 & Marcapata* \\
\hline Cicadellinae & Cicadellini & Eryhrogonia eburata* & -71.033 & -14.483 & 4240 & Vilcanota* \\
\hline Cicadellinae & Cicadellini & Erythrogonia imitatricula* & -71.540 & -12.820 & 1500 & Callanga* \\
\hline Cicadellinae & Cicadellini & Erythrogonia imitatricula* & -71.550 & -13.060 & 1590 & Santa Isabel ${ }^{*}$ \\
\hline Cicadellinae & Cicadellini & Erythrogonia kokomona* & -70.894 & -13.503 & 1880 & Marcapata* \\
\hline Cicadellinae & Cicadellini & Erythrogonia socialis* & -70.894 & -13.503 & 1880 & Marcapata* \\
\hline Cicadellinae & Cicadellini & Erythrogonia triplicula* & -70.894 & -13.503 & 1880 & Marcapata* \\
\hline Cicadellinae & Cicadellini & Erythrogonia triplicula & -71.430 & -12.980 & 565 & Pilcopata \\
\hline
\end{tabular}


Apéndice 1. Continuación.

\begin{tabular}{|c|c|c|c|c|c|c|}
\hline Subfamilia & Tribu & Especie & Longitud & Latitud & Altitud & Localidad \\
\hline Cicadellinae & Cicadellini & Erythrogonia triplicula & -72.550 & -13.170 & 2450 & Machupicchu* \\
\hline Cicadellinae & Cicadellini & Erythrogonia warsula* & -70.894 & -13.503 & 1880 & Marcapata* \\
\hline Cicadellinae & Cicadellini & Hortensia similis & -72.510 & -12.680 & 800 & Sahuayaco \\
\hline Cicadellinae & Cicadellini & Hortensia similis & -71.430 & -12.980 & 565 & Pilcopata \\
\hline Cicadellinae & Cicadellini & Iragua montana* & -71.033 & -14.483 & 4240 & Vilcanota* \\
\hline Cicadellinae & Cicadellini & Iragua perplexa* & -70.894 & -13.503 & 1880 & Marcapata* \\
\hline Cicadellinae & Cicadellini & Jakrama eureta* & -71.540 & -12.820 & 1500 & Callanga* \\
\hline Cicadellinae & Cicadellini & Jozima leucopa & -71.300 & -12.940 & 790 & Atalaya \\
\hline Cicadellinae & Cicadellini & Jozima leucopa* & -70.894 & -13.503 & 1880 & Marcapata* \\
\hline Cicadellinae & Cicadellini & Juliaca bilineata* & -71.540 & -12.820 & 1500 & Callanga* \\
\hline Cicadellinae & Cicadellini & Juliaca simoni* & -71.550 & -13.060 & 1590 & Santa Isabel* \\
\hline Cicadellinae & Cicadellini & Juliaca simoni* & -71.540 & -12.820 & 1500 & Callanga* \\
\hline Cicadellinae & Cicadellini & Korigonalia cajana* & -71.540 & -12.820 & 1500 & Callanga* \\
\hline Cicadellinae & Cicadellini & Lissoscarta nipata* & -71.390 & -12.910 & 800 & Hacienda María* \\
\hline Cicadellinae & Cicadellini & Macugonalia cavifrons & -71.300 & -12.940 & 790 & Atalaya \\
\hline Cicadellinae & Cicadellini & Macugonalia leucomelas & -71.430 & -12.980 & 565 & Pilcopata \\
\hline Cicadellinae & Cicadellini & Mesogonia callangana* & -71.540 & -12.820 & 1500 & Callanga* \\
\hline Cicadellinae & Cicadellini & Mesogonia callangana & -71.300 & -12.940 & 790 & Atalaya \\
\hline Cicadellinae & Cicadellini & Mesogonia olivatula & -71.300 & -12.940 & 790 & Atalaya \\
\hline Cicadellinae & Cicadellini & Mesogonia retrorsa* & -71.540 & -12.820 & 1500 & Callanga* \\
\hline Cicadellinae & Cicadellini & Mesogonia suspecta* & -71.597 & -13.321 & 3000 & Paucartambo* \\
\hline Cicadellinae & Cicadellini & Mesogonia tolosa* & -71.430 & -12.980 & 565 & Pilcopata* \\
\hline Cicadellinae & Cicadellini & Mucrometopia caudata* & -71.033 & -14.483 & 4240 & Vilcanota* \\
\hline Cicadellinae & Cicadellini & Onega bracteata & -72.510 & -12.680 & 800 & Sahuayaco \\
\hline Cicadellinae & Cicadellini & Onega bracteata & -71.430 & -12.980 & 565 & Pilcopata \\
\hline Cicadellinae & Cicadellini & Onega bracteata* & -71.540 & -12.820 & 1500 & Callanga* \\
\hline Cicadellinae & Cicadellini & Onega sp & -71.560 & -13.100 & 1400 & San Pedro \\
\hline Cicadellinae & Cicadellini & Onega sp & -71.590 & -13.140 & 2400 & Paucart-Pilcopata \\
\hline Cicadellinae & Cicadellini & Oragua nusinasa* & -71.550 & -13.060 & 1590 & Santa Isabel ${ }^{*}$ \\
\hline Cicadellinae & Cicadellini & Oragua nusinasa* & -70.894 & -13.503 & 1880 & Marcapata* \\
\hline Cicadellinae & Cicadellini & Oragua nusinasa* & -71.540 & -12.820 & 1500 & Callanga* \\
\hline Cicadellinae & Cicadellini & Oragua partitula & -71.430 & -12.980 & 565 & Pilcopata \\
\hline Cicadellinae & Cicadellini & Pachitea habernula* & -71.540 & -12.820 & 1500 & Callanga* \\
\hline Cicadellinae & Cicadellini & Pachitea ryma & -71.569 & -13.105 & 1300 & Paucart-Pilcopata \\
\hline Cicadellinae & Cicadellini & Pachitea ryma & -71.430 & -13.170 & 950 & Paucart-Pilcopata \\
\hline Cicadellinae & Cicadellini & Pachitea ryma* & -71.540 & -12.820 & 1500 & Callanga* \\
\hline Cicadellinae & Cicadellini & Pachitea subflava & -71.430 & -13.170 & 950 & Paucart-Pilcopata \\
\hline Cicadellinae & Cicadellini & Pamplona spatulata* & -71.540 & -12.820 & 1500 & Callanga* \\
\hline Cicadellinae & Cicadellini & Parinaeota bakeri* & -70.894 & -13.503 & 1880 & Marcapata* \\
\hline Cicadellinae & Cicadellini & Paromenia marginata & -71.500 & -13.210 & 1130 & Paucart-Pilcopata \\
\hline Cicadellinae & Cicadellini & Paromenia marginata* & -71.550 & -13.060 & 1590 & Santa Isabel ${ }^{*}$ \\
\hline Cicadellinae & Cicadellini & Paromenia quimbayensis & -71.600 & -13.120 & 2750 & Trocha Unión \\
\hline Cicadellinae & Cicadellini & Paromenia quimbayensis & -71.430 & -12.980 & 565 & Pilcopata \\
\hline Cicadellinae & Cicadellini & Paromenia quimbayensis & -71.580 & -13.150 & 2900 & Esperanza \\
\hline Cicadellinae & Cicadellini & Paromenia quimbayensis & -71.790 & -13.120 & 2628 & Pillahuata \\
\hline Cicadellinae & Cicadellini & Pawiloma fulpae & -72.550 & -13.170 & 2450 & Machupicchu* \\
\hline Cicadellinae & Cicadellini & Pawiloma fulpae & -72.886 & -13.409 & 1600 & Ucchuhuerta \\
\hline Cicadellinae & Cicadellini & Pawiloma sirochia & -71.600 & -13.120 & 2750 & Trocha Unión \\
\hline Cicadellinae & Cicadellini & Punahuana dyscrita & -71.600 & -13.120 & 2750 & Trocha Unión \\
\hline Cicadellinae & Cicadellini & Punahuana dyscrita* & -70.894 & -13.503 & 1880 & Marcapata* \\
\hline Cicadellinae & Cicadellini & Punahuana dyscrita* & -71.540 & -12.820 & 1500 & Callanga* \\
\hline Cicadellinae & Cicadellini & Ramosulus corrugipennis & -71.430 & -12.980 & 565 & Pilcopata \\
\hline Cicadellinae & Cicadellini & Ramosulus phaedrus & -71.600 & -13.120 & 2750 & Trocha Unión \\
\hline Cicadellinae & Cicadellini & Schildola ductilis* & -71.550 & -13.060 & 1590 & Santa Isabel* \\
\hline Cicadellinae & Cicadellini & Sibovia picchitula* & -72.550 & -13.170 & 2450 & Machupicchu* \\
\hline Cicadellinae & Cicadellini & Sibovia praevia* & -71.980 & -13.510 & 3400 & Cusco* \\
\hline Cicadellinae & Cicadellini & Sibovia aprica & -72.150 & -12.810 & 1618 & Miraflores \\
\hline Cicadellinae & Cicadellini & Soosiulus flammidulus* & -70.894 & -13.503 & 1880 & Marcapata* \\
\hline Cicadellinae & Cicadellini & Soosiulus seimunculus* & -70.894 & -13.503 & 1880 & Marcapata* \\
\hline Cicadellinae & Cicadellini & Stehlikiana halticula & -72.550 & -13.170 & 2450 & Machupicchu* \\
\hline Cicadellinae & Cicadellini & Stehlikiana halticula & -71.430 & -12.980 & 565 & Pilcopata \\
\hline Cicadellinae & Cicadellini & Stehlikiana halticula & -71.590 & -13.160 & 2400 & Paucart-Pilcopata \\
\hline Cicadellinae & Cicadellini & Tipuana expallida* & -71.390 & -12.910 & 800 & Hacienda María* \\
\hline Cicadellinae & Cicadellini & Tortigonalia torta* & -71.540 & -12.820 & 1500 & Callanga* \\
\hline Cicadellinae & Cicadellini & Tortigonalia treva & -71.430 & -12.980 & 565 & Pilcopata \\
\hline Cicadellinae & Cicadellini & Tortigonalia treva* & -71.540 & -12.820 & 1500 & Callanga* \\
\hline Cicadellinae & Cicadellini & Trichogonia costata & -71.960 & -13.520 & 3320 & Ttio \\
\hline
\end{tabular}

(Continúa...) 
Apéndice 1. Continuación.

\begin{tabular}{|c|c|c|c|c|c|c|}
\hline Subfamilia & Tribu & Especie & Longitud & Latitud & Altitud & Localidad \\
\hline Cicadellinae & Cicadellini & Trichogonia costata & -71.580 & -13.530 & 3380 & Huancaro \\
\hline Cicadellinae & Cicadellini & Trichogonia costata & -71.980 & -13.500 & 3600 & Saqsayhuaman \\
\hline Cicadellinae & Cicadellini & Trichogonia costata & -71.990 & -13.510 & 3520 & Ticatica \\
\hline Cicadellinae & Cicadellini & Trichogonia costata & -71.940 & -13.510 & 3340 & Salineras \\
\hline Cicadellinae & Cicadellini & Trichogonia costata & -71.890 & -13.560 & 3270 & Pillao Matao \\
\hline Cicadellinae & Cicadellini & Trichogonia costata & -71.980 & -13.510 & 3400 & Cusco \\
\hline Cicadellinae & Cicadellini & Trichogonia costata & -71.840 & -13.040 & 3340 & Pampallaqta \\
\hline Cicadellinae & Cicadellini & Trichogonia costata & -71.650 & -13.620 & 3600 & Churubamba \\
\hline Cicadellinae & Cicadellini & Zaruma decolorata* & -71.540 & -12.820 & 1500 & Callanga* \\
\hline Cicadellinae & Proconiini & Abana horvathi* & -71.980 & -13.510 & 3400 & Cusco* \\
\hline Cicadellinae & Proconiini & Acrogonia stylata* & -71.390 & -12.910 & 800 & Hacienda María* \\
\hline Cicadellinae & Proconiini & Acrogonia stylata* & -71.540 & -12.820 & 1500 & Callanga* \\
\hline Cicadellinae & Proconiini & Anacuerna centrolinea & -71.940 & -13.560 & 3100 & Tipón \\
\hline Cicadellinae & Proconiini & Anacuerna centrolinea & -71.820 & -13.560 & 3150 & Saylla \\
\hline Cicadellinae & Proconiini & Anacuerna centrolinea & -71.959 & -13.522 & 3340 & Perayoc \\
\hline Cicadellinae & Proconiini & Anacuerna centrolinea & -71.990 & -13.510 & 3520 & Ticatica \\
\hline Cicadellinae & Proconiini & Anacuerna centrolinea & -71.920 & -13.510 & 3580 & Pumamarca \\
\hline Cicadellinae & Proconiini & Anacuerna centrolinea & -71.890 & -13.560 & 3280 & Pillao Matao \\
\hline Cicadellinae & Proconiini & Anacuerna centrolinea & -71.870 & -13.540 & 3210 & Kayra \\
\hline Cicadellinae & Proconiini & Deselvana longipennis & -71.430 & -12.980 & 565 & Pilcopata \\
\hline Cicadellinae & Proconiini & Dicrophleps truncata & -71.300 & -12.940 & 790 & Atalaya \\
\hline Cicadellinae & Proconiini & Dicrophleps truncata* & -71.390 & -12.910 & 800 & Hacienda María* \\
\hline Cicadellinae & Proconiini & Dicrophleps truncata* & -71.360 & -13.000 & 540 & Río Kosñipata* \\
\hline Cicadellinae & Proconiini & Diestostemma blantoni* & -71.540 & -12.820 & 1500 & Callanga* \\
\hline Cicadellinae & Proconiini & Diestostemma dolosum* & -71.980 & -13.510 & 3400 & Cusco* \\
\hline Cicadellinae & Proconiini & Diestostemma reticulatum* & -71.980 & -13.510 & 3400 & Cusco* \\
\hline Cicadellinae & Proconiini & Egidemia obtusata* & -70.894 & -13.503 & 1880 & Marcapata* \\
\hline Cicadellinae & Proconiini & Homoscarta boliviana & -71.430 & -12.980 & 565 & Pilcopata \\
\hline Cicadellinae & Proconiini & Homoscarta superciliaris* & -71.980 & -13.510 & 3400 & Cusco* \\
\hline Cicadellinae & Proconiini & Ichthyobelus bellicosus & -72.510 & -12.680 & 800 & Sahuayaco \\
\hline Cicadellinae & Proconiini & Molomea consorta* & -71.020 & -14.480 & 4240 & Vilcanota* \\
\hline Cicadellinae & Proconiini & Molomea guttulata & -72.510 & -12.680 & 800 & Sahuayaco \\
\hline Cicadellinae & Proconiini & Oncometopia venata & -71.430 & -12.980 & 565 & Pilcopata \\
\hline Cicadellinae & Proconiini & Oncometopia venata* & -71.249 & -12.684 & 441 & Shintuya* \\
\hline Cicadellinae & Proconiini & Oncometopia sp & -71.430 & -12.980 & 565 & Pilcopata \\
\hline Cicadellinae & Proconiini & Oncometopia sp & -72.510 & -12.680 & 800 & Sahuayaco \\
\hline Cicadellinae & Proconiini & Oncometopia sp & -71.300 & -12.940 & 790 & Atalaya \\
\hline Cicadellinae & Proconiini & Oncometopia sp & -71.430 & -13.170 & 950 & Paucart-Pilcopata \\
\hline Cicadellinae & Proconiini & Procandea andina* & -70.894 & -13.503 & 1880 & Marcapata* \\
\hline Cicadellinae & Proconiini & Procandea cordillerae* & -71.980 & -13.510 & 3400 & Cusco* \\
\hline Cicadellinae & Proconiini & Procandea inca & -71.430 & -12.980 & 565 & Pilcopata \\
\hline Cicadellinae & Proconiini & Procandea monticola* & -71.550 & -13.060 & 1590 & Santa Isabel* \\
\hline Cicadellinae & Proconiini & Procandea monticola* & -71.540 & -12.820 & 1500 & Callanga* \\
\hline Cicadellinae & Proconiini & Procandea quechua* & -71.540 & -12.820 & 1500 & Callanga* \\
\hline Cicadellinae & Proconiini & Procandea quechua* & -71.980 & -13.510 & 3400 & Cusco* \\
\hline Cicadellinae & Proconiini & Proconopera pullula* & -71.540 & -12.820 & 1500 & Callanga* \\
\hline Cicadellinae & Proconiini & Proconosama haenschi & -72.510 & -12.680 & 800 & Sahuayaco \\
\hline Cicadellinae & Proconiini & Proconosama haenschi & -71.560 & -13.100 & 1400 & San Pedro \\
\hline Cicadellinae & Proconiini & Proconia marmorata & -71.430 & -12.980 & 565 & Pilcopata \\
\hline Cicadellinae & Proconiini & Pseudometopia irenae & -71.560 & -13.100 & 1400 & San Pedro \\
\hline Cicadellinae & Proconiini & Pseudometopia irenae* & -71.550 & -13.060 & 1590 & Santa Isabel* \\
\hline Cicadellinae & Proconiini & Pseudometopia irenae* & -71.540 & -12.820 & 1500 & Callanga* \\
\hline Cicadellinae & Proconiini & $\begin{array}{l}\text { Rhaphirrhinus } \\
\text { phosphoreus }\end{array}$ & -71.430 & -12.980 & 565 & Pilcopata \\
\hline Deltocephalinae & Deltocephalini & Amplicephalus auranticus* & -72.550 & -13.170 & 2450 & Machupicchu* \\
\hline Deltocephalinae & Deltocephalini & Amplicephalus paradoxus* & -72.550 & -13.170 & 2450 & Machupicchu* \\
\hline Deltocephalinae & Deltocephalini & Mattogrossus colonoides* & -70.740 & -13.220 & 690 & Quince Mil* \\
\hline Deltocephalinae & Deltocephalini & Picchuia pungens* & -72.550 & -13.170 & 2450 & Machupicchu* \\
\hline Deltocephalinae & Doraturini & Icaia appendiculata & -72.050 & -13.390 & 3720 & Huatata \\
\hline Deltocephalinae & Doraturini & Icaia appendiculata* & -72.550 & -13.170 & 2450 & Machupicchu* \\
\hline Deltocephalinae & Euscelini & Andanus bimaculatus* & -70.740 & -13.210 & 690 & Quince Mil \\
\hline Deltocephalinae & Euscelini & Atanus picchuanus* & -72.550 & -13.170 & 2450 & Machupicchu* \\
\hline Deltocephalinae & Euscelini & Bandaromimus fulvopictus & -71.300 & -12.940 & 790 & Atalaya \\
\hline Deltocephalinae & Euscelini & Chlorotettix sp & -71.600 & -13.120 & 2750 & Trocha Unión \\
\hline Deltocephalinae & Euscelini & Exitianus quadratulus* & -72.550 & -13.170 & 2450 & Machupicchu* \\
\hline Deltocephalinae & Euscelini & Paratanus exitiosus & -72.260 & -13.250 & 2842 & Ollantaytambo \\
\hline Deltocephalinae & Euscelini & Paratanus exitiosus & -72.280 & -13.250 & 2800 & Chilca \\
\hline Deltocephalinae & Euscelini & Paratanus exitiosus & -72.080 & -13.090 & 3545 & Cuncani \\
\hline
\end{tabular}

(Continúa...) 
Apéndice 1. Continuación.

\begin{tabular}{|c|c|c|c|c|c|c|}
\hline Subfamilia & Tribu & Especie & Longitud & Latitud & Altitud & Localidad \\
\hline Deltocephalinae & Euscelini & Paratanus exitiosus & -72.070 & -13.110 & 3510 & Tambohuaylla \\
\hline Deltocephalinae & Euscelini & Paratanus exitiosus & -71.230 & -14.250 & 3600 & Marangani \\
\hline Deltocephalinae & Euscelini & Paratanus exitiosus & -71.380 & -14.166 & 3500 & Sicuani \\
\hline Deltocephalinae & Euscelini & Paratanus exitiosus & -71.870 & -13.540 & 3210 & Kayra \\
\hline Deltocephalinae & Euscelini & Paratanus exitiosus & -71.140 & -14.380 & 3600 & Mamuera \\
\hline Deltocephalinae & Euscelini & Paratanus exitiosus & -72.110 & -13.370 & 3600 & Cruzpata \\
\hline Deltocephalinae & Euscelini & Paratanus yusti & -71.980 & -13.500 & 3600 & Saqsayhuaman \\
\hline Deltocephalinae & Euscelini & Paratanus yusti & -71.940 & -13.540 & 3350 & Tancarpata \\
\hline Deltocephalinae & Euscelini & Paratanus yusti & -71.880 & -13.540 & 3240 & San Jerónimo \\
\hline Deltocephalinae & Euscelini & Paratanus yusti & -71.950 & -13.520 & 3340 & Perayoc \\
\hline Deltocephalinae & Euscelini & Paratanus yusti & -71.980 & -13.520 & 3430 & Santiago \\
\hline Deltocephalinae & Euscelini & Paratanus yusti & -71.920 & -13.510 & 3580 & Pumamarca \\
\hline Deltocephalinae & Euscelini & Paratanus yusti & -71.930 & -13.530 & 3290 & San Sebastián \\
\hline Deltocephalinae & Euscelini & Paratanus yusti & -71.870 & -13.540 & 3210 & Kayra \\
\hline Deltocephalinae & Euscelini & Paratanus yusti & -72.440 & -13.470 & 2530 & Limatambo \\
\hline Deltocephalinae & Euscelini & Paratanus yusti & -71.960 & -13.520 & 3320 & Wanchaq \\
\hline Deltocephalinae & Euscelini & Paratanus yusti & -71.890 & -13.510 & 3450 & Santa Maria \\
\hline Deltocephalinae & Euscelini & Paratanus yusti & -72.260 & -13.250 & 2842 & Ollantaytambo \\
\hline Deltocephalinae & Euscelini & Paratanus yusti & -72.280 & -13.250 & 2800 & Chilca \\
\hline Deltocephalinae & Euscelini & Paratanus yusti & -72.120 & -13.300 & 2925 & Urubamba \\
\hline Deltocephalinae & Euscelini & Paratanus yusti & -71.140 & -14.380 & 3600 & Mamuera \\
\hline Deltocephalinae & Euscelini & Sinchonoa machua* & -72.550 & -13.170 & 2450 & Machupicchu* \\
\hline Deltocephalinae & Euscelini & Yungasia longipennis* & -72.550 & -13.170 & 2450 & Machupicchu* \\
\hline Deltocephalinae & Hecalini & Tenucephalus saggitarius* & -72.550 & -13.170 & 2450 & Machupicchu* \\
\hline Deltocephalinae & Macrostelini & Dalbulus maidis & -72.260 & -13.250 & 2842 & Ollantaytambo \\
\hline Deltocephalinae & Macrostelini & Picchusteles inca* & -72.550 & -13.170 & 2450 & Machu Pichu* \\
\hline Deltocephalinae & Scaphytopiini & Scaphytopius atrifrons* & -72.550 & -13.170 & 2450 & Machu Pichu* \\
\hline Deltocephalinae & Tribu Incierta & Amblysellus sp & -71.880 & -13.540 & 3240 & San Jerónimo \\
\hline Deltocephalinae & Tribu Incierta & Amblysellus sp & -71.950 & -13.520 & 3340 & Perayoc \\
\hline Deltocephalinae & Tribu Incierta & Amblysellus sp & -71.940 & -13.540 & 3350 & Tancarpata \\
\hline Deltocephalinae & Tribu Incierta & Amblysellus sp & -71.980 & -13.520 & 3430 & Santiago \\
\hline Deltocephalinae & Tribu Incierta & Amblysellus sp & -72.000 & -13.150 & 3730 & Pampacorral \\
\hline Deltocephalinae & Tribu Incierta & Amblysellus sp & -71.580 & -13.150 & 2900 & Esperanza \\
\hline Deltocephalinae & Tribu Incierta & Amblysellus sp & -72.080 & -13.090 & 3545 & Cuncani \\
\hline Deltocephalinae & Tribu Incierta & Amblysellus sp & -72.070 & -13.110 & 3510 & Tambohuaylla \\
\hline Deltocephalinae & Tribu Incierta & Amblysellus sp & -72.450 & -13.480 & 2530 & Limatambo \\
\hline Deltocephalinae & Tribu Incierta & Bahita infuscata & -71.430 & -12.980 & 565 & Pilcopata \\
\hline Deltocephalinae & Tribu Incierta & Bahita infuscata & -71.500 & -13.210 & 1130 & Paucart-Pilcopata \\
\hline Deltocephalinae & Tribu Incierta & Bahita infuscata & -71.300 & -12.940 & 790 & Atalaya \\
\hline Deltocephalinae & Tribu Incierta & Bahita irrorata & -71.500 & -13.210 & 1130 & Paucart-Pilcopata \\
\hline Deltocephalinae & Tribu Incierta & Bahita irrorata & -71.430 & -13.170 & 950 & Paucart-Pilcopata \\
\hline Deltocephalinae & Tribu Incierta & Bahita sp & -71.600 & -13.120 & 2750 & Trocha Unión \\
\hline Deltocephalinae & Tribu Incierta & Haldorus sp & -71.600 & -13.120 & 2750 & Trocha Unión \\
\hline Deltocephalinae & Tribu Incierta & Neomesus sp & -71.980 & -13.500 & 3600 & Saqsayhuaman \\
\hline Deltocephalinae & Tribu Incierta & Neomesus sp & -72.050 & -13.390 & 3720 & Huatata \\
\hline Iassinae & Gyponini & Acuera nana & -71.600 & -13.120 & 2750 & Trocha Unión \\
\hline Iassinae & Gyponini & Acuera nana & -71.300 & -12.940 & 790 & Atalaya \\
\hline Iassinae & Gyponini & Acuera nana* & -71.360 & -13.000 & 540 & Río Kosñipata* \\
\hline Iassinae & Gyponini & Acuera sp & -71.430 & -13.170 & 950 & Paucart-Pilcopata \\
\hline Iassinae & Gyponini & Acuera sp & -71.300 & -12.940 & 790 & Atalaya \\
\hline Iassinae & Gyponini & Acuera sp & -71.500 & -13.210 & 1130 & Paucart-Pilcopata \\
\hline Iassinae & Gyponini & Acuponana enera* & -71.390 & -12.910 & 800 & Hacienda María* \\
\hline Iassinae & Gyponini & Barbatana narda* & -71.550 & -13.060 & 1590 & Santa Isabel ${ }^{*}$ \\
\hline Iassinae & Gyponini & Chloronana decolora* & -71.390 & -12.910 & 800 & Hacienda María* \\
\hline Iassinae & Gyponini & Chloronana orbicula & -71.300 & -12.940 & 790 & Atalaya \\
\hline Iassinae & Gyponini & Chloronana rotunda* & -71.540 & -12.820 & 1500 & Callanga* \\
\hline Iassinae & Gyponini & Costanana sp 1 & -71.950 & -13.520 & 3340 & Perayoc \\
\hline Iassinae & Gyponini & Costanana sp 2 & -71.600 & -13.120 & 2750 & Trocha Unión \\
\hline Iassinae & Gyponini & Costanana sp 2 & -71.500 & -13.210 & 1130 & Paucart-Pilcopata \\
\hline Iassinae & Gyponini & Costanana sp 2 & -71.430 & -13.170 & 950 & Paucart-Pilcopata \\
\hline Iassinae & Gyponini & Costanana sp 2 & -71.590 & -13.140 & 2400 & Paucart-Pilcopata \\
\hline Iassinae & Gyponini & Culumana bacula* & -72.550 & -13.170 & 2450 & Machupicchu* \\
\hline Iassinae & Gyponini & Culumana concava* & -71.550 & -13.060 & 1590 & Santa Isabel* \\
\hline Iassinae & Gyponini & Culumana dualana* & -71.550 & -13.060 & 1590 & Santa Isabel* \\
\hline Iassinae & Gyponini & Curtara picchua* & -72.550 & -13.170 & 2450 & Machupicchu* \\
\hline Iassinae & Gyponini & Curtara sufflara* & -71.540 & -12.820 & 1500 & Callanga* \\
\hline Iassinae & Gyponini & Folicana nota* & -71.390 & -12.910 & 800 & Hacienda María* \\
\hline Iassinae & Gyponini & Folicana nota* & -71.540 & -12.820 & 1500 & Callanga* $^{*}$ \\
\hline
\end{tabular}


Apéndice 1. Continuación.

\begin{tabular}{|c|c|c|c|c|c|c|}
\hline Subfamilia & Tribu & Especie & Longitud & Latitud & Altitud & Localidad \\
\hline Iassinae & Gyponini & Fuminana astra* & -71.540 & -12.820 & 1500 & Callanga* \\
\hline Iassinae & Gyponini & Fuminana lira* & -71.390 & -12.910 & 800 & Hacienda María* \\
\hline Iassinae & Gyponini & Gypona adita* & -71.550 & -13.060 & 1590 & Santa Isabel* \\
\hline Iassinae & Gyponini & Gypona fisura* & -71.390 & -12.910 & 800 & Hacienda María* \\
\hline Iassinae & Gyponini & Gypona glauca & -71.600 & -13.120 & 2750 & Trocha Unión \\
\hline Iassinae & Gyponini & Gypona nacula* & -71.390 & -12.910 & 800 & Hacienda María* \\
\hline Iassinae & Gyponini & Gypona nigrena* & -71.540 & -12.820 & 1500 & Callanga* \\
\hline Iassinae & Gyponini & Gypona quadrina* & -72.550 & -13.170 & 2450 & Machupicchu* \\
\hline Iassinae & Gyponini & Gypona quadrina & -71.430 & -12.980 & 565 & Pilcopata \\
\hline Iassinae & Gyponini & Gypona solata* & -71.540 & -12.820 & 1500 & Callanga* \\
\hline Iassinae & Gyponini & Gyponana alicata & -71.430 & -12.980 & 565 & Pilcopata \\
\hline Iassinae & Gyponini & Gyponana alicata & -71.300 & -12.940 & 790 & Atalaya \\
\hline Iassinae & Gyponini & Hecalapona apicella* & -71.540 & -12.820 & 1500 & Callanga* \\
\hline Iassinae & Gyponini & Hecalapona berna* & -71.540 & -12.820 & 1500 & Callanga* \\
\hline Iassinae & Gyponini & Hecalapona crinata* & -71.540 & -12.820 & 1500 & Callanga* \\
\hline Iassinae & Gyponini & Hecalapona eruva* & -70.740 & -13.220 & 690 & Quince Mil* \\
\hline Iassinae & Gyponini & Hecalapona forceps* & -71.540 & -12.820 & 1500 & Callanga* \\
\hline Iassinae & Gyponini & Hecalapona huella* & -71.540 & -12.820 & 1500 & Callanga* \\
\hline Iassinae & Gyponini & Hecalapona scella* & -71.540 & -12.820 & 1500 & Callanga* \\
\hline Iassinae & Gyponini & Nullana sinchona* & -71.550 & -13.060 & 1590 & Santa Isabel* \\
\hline Iassinae & Gyponini & Nullana verdana* & -71.390 & -12.910 & 800 & Hacienda María* \\
\hline Iassinae & Gyponini & Polana bulba* & -71.540 & -12.820 & 1500 & Callanga* \\
\hline Iassinae & Gyponini & Polana concinna* & -71.540 & -12.820 & 1500 & Callanga* \\
\hline Iassinae & Gyponini & Polana falsa* & -71.390 & -12.910 & 800 & Hacienda María* \\
\hline Iassinae & Gyponini & Polana fetera* & -71.390 & -12.910 & 800 & Hacienda María* \\
\hline Iassinae & Gyponini & Polana fetera* & -71.550 & -13.060 & 1590 & Santa Isabel* \\
\hline Iassinae & Gyponini & Polana icara & -71.500 & -13.210 & 1130 & Paucart-Pilcopata \\
\hline Iassinae & Gyponini & Polana icara* & -71.550 & -13.060 & 1590 & Santa Isabel* \\
\hline Iassinae & Gyponini & Polana inimicus* & -71.540 & -12.820 & 1500 & Callanga* \\
\hline Iassinae & Gyponini & Polana lanara* & -71.550 & -13.060 & 1590 & Santa Isabel* \\
\hline Iassinae & Gyponini & Polana lanara* & -71.390 & -12.910 & 800 & Hacienda María* \\
\hline Iassinae & Gyponini & Polana resupina* & -71.390 & -12.910 & 800 & Hacienda María* \\
\hline Iassinae & Gyponini & Polana scruta* & -71.390 & -12.910 & 800 & Hacienda María* \\
\hline Iassinae & Gyponini & Polana sp & -71.500 & -13.210 & 1130 & Paucart-Pilcopata \\
\hline Iassinae & Gyponini & Polana sp & -71.430 & -13.170 & 950 & Paucart-Pilcopata \\
\hline Iassinae & Gyponini & Polana sp & -71.580 & -13.150 & 2900 & Esperanza \\
\hline Iassinae & Gyponini & Polana sp & -71.790 & -13.120 & 2628 & Pillahuata \\
\hline Iassinae & Gyponini & Ponana atea* & -71.550 & -13.060 & 1590 & Santa Isabel* \\
\hline Iassinae & Gyponini & Ponana avena* & -71.550 & -13.060 & 1590 & Santa Isabel* \\
\hline Iassinae & Gyponini & Ponana berta* & -71.550 & -13.060 & 1590 & Santa Isabel ${ }^{*}$ \\
\hline Iassinae & Gyponini & Ponana cerosa* & -71.790 & -13.120 & 2628 & Pillahuata* \\
\hline Iassinae & Gyponini & Ponana cerosa* & -71.597 & -13.320 & 2977 & Paucartambo* \\
\hline Iassinae & Gyponini & Ponana hilara* & -71.550 & -13.060 & 1590 & Santa Isabel* \\
\hline Iassinae & Gyponini & Ponana perusana* & -71.550 & -13.060 & 1590 & Santa Isabel* \\
\hline Iassinae & Gyponini & Ponana sp & -71.600 & -13.120 & 2750 & Trocha Unión \\
\hline Iassinae & Gyponini & Ponana sp & -71.590 & -13.160 & 2400 & Paucart-Pilcopata \\
\hline Iassinae & Gyponini & Ponana sp & -71.500 & -13.210 & 1130 & Paucart-Pilcopata \\
\hline Iassinae & Gyponini & Ponana sp & -71.430 & -13.170 & 950 & Paucart-Pilcopata \\
\hline Iassinae & Gyponini & Ponanella rubravenosa* & -71.540 & -12.820 & 1500 & Callanga* \\
\hline Iassinae & Gyponini & Scaris amputa* & -71.390 & -12.910 & 800 & Hacienda María* \\
\hline Iassinae & Gyponini & Scaris caballa* & -71.390 & -12.910 & 800 & Hacienda María* \\
\hline Iassinae & Gyponini & Scaris defecta* & -72.139 & -13.301 & 2900 & Río Urubamba* \\
\hline Iassinae & Gyponini & Scaris fimbriella* & -71.390 & -12.910 & 800 & Hacienda María* \\
\hline Iassinae & Gyponini & Scaris maculosa* & -71.550 & -13.060 & 1590 & Santa Isabel* \\
\hline Iassinae & Gyponini & Scaris serosa & -71.300 & -12.940 & 790 & Atalaya \\
\hline Iassinae & Gyponini & Scaris serosa & -71.350 & -12.850 & 300 & Santa Cruz \\
\hline Iassinae & Gyponini & Tenuacia rubera* & -71.550 & -13.060 & 1590 & Santa Isabel* \\
\hline Iassinae & Gyponini & Tenuacia rubera* & -71.390 & -12.910 & 800 & Hacienda María* \\
\hline Iassinae & Gyponini & Tuberana tubera & -71.430 & -12.980 & 565 & Pilcopata \\
\hline Iassinae & Iassini & Grunchia grossa* & -71.540 & -12.820 & 1500 & Callanga* \\
\hline Ledrinae & Xerophloeini & Xerophloea viridis & -72.440 & -13.470 & 2530 & Limatambo \\
\hline Megophthalminae & Agalliini & Agallia sp & -72.510 & -12.680 & 800 & Sahuayaco \\
\hline Megophthalminae & Agalliini & Agallia sp & -71.500 & -13.210 & 1130 & Paucart-Pilcopata \\
\hline Megophthalminae & Agalliini & Agallia sp & -71.300 & -12.940 & 790 & Atalaya \\
\hline Megophthalminae & Agalliini & Agallia sp & -71.600 & -13.120 & 2750 & Trocha Unión \\
\hline Megophthalminae & Agalliini & Agallia sp & -71.580 & -13.150 & 2900 & Esperanza \\
\hline Megophthalminae & Agalliini & Agallia sp & -71.790 & -13.120 & 2628 & Pillahuata \\
\hline Megophthalminae & Agalliini & Agalliopsis atahualpa* & -72.550 & -13.170 & 2450 & Machupicchu* \\
\hline
\end{tabular}


Apéndice 1. Continuación.

\begin{tabular}{|c|c|c|c|c|c|c|}
\hline Subfamilia & Tribu & Especie & Longitud & Latitud & Altitud & Localidad \\
\hline Megophthalminae & Agalliini & Agalliopsis bicuspidata* & -72.550 & -13.170 & 2450 & Machupicchu* \\
\hline Megophthalminae & Agalliini & Agalliopsis cervina & -71.430 & -13.170 & 950 & Paucart-Pilcopata \\
\hline Megophthalminae & Agalliini & Agalliopsis peruviana* & -71.540 & -12.820 & 1500 & Callanga* \\
\hline Megophthalminae & Agalliini & Agalliopsis spinosa* & -72.550 & -13.170 & 2450 & Machupicchu* \\
\hline Megophthalminae & Agalliini & Agalliopsis vittata* & -72.550 & -13.170 & 2450 & Machupicchu* \\
\hline Typhlocybinae & Empoascini & Empoasca sp & -71.980 & -13.500 & 3600 & Saqsayhuaman \\
\hline Typhlocybinae & Empoascini & Empoasca sp & -71.940 & -13.540 & 3350 & Tancarpata \\
\hline Typhlocybinae & Empoascini & Empoasca sp & -71.920 & -13.510 & 3580 & Pumamarca \\
\hline Typhlocybinae & Empoascini & Empoasca sp & -71.930 & -13.530 & 3290 & San Sebastián \\
\hline Typhlocybinae & Empoascini & Empoasca sp & -71.960 & -13.520 & 3320 & Wanchaq \\
\hline Typhlocybinae & Empoascini & Empoasca sp & -71.890 & -13.510 & 3450 & Santa Maria \\
\hline Typhlocybinae & Empoascini & Empoasca sp & -71.820 & -13.560 & 3150 & Saylla \\
\hline Typhlocybinae & Empoascini & Empoasca sp & -71.430 & -12.980 & 565 & Pilcopata \\
\hline Typhlocybinae & Empoascini & Empoasca sp & -71.580 & -13.150 & 2900 & Esperanza \\
\hline Typhlocybinae & Empoascini & Empoasca sp & -71.790 & -13.120 & 2628 & Pillahuata \\
\hline Typhlocybinae & Empoascini & Empoasca sp & -71.960 & -13.520 & 3320 & Ttio \\
\hline Typhlocybinae & Empoascini & Empoasca sp & -71.580 & -13.530 & 3380 & Huancaro \\
\hline Typhlocybinae & Empoascini & Empoasca sp & -71.990 & -13.510 & 3520 & Ticatica \\
\hline Typhlocybinae & Empoascini & Empoasca sp & -71.940 & -13.510 & 3340 & Salineras \\
\hline Typhlocybinae & Empoascini & Empoasca sp & -71.890 & -13.560 & 3270 & Pillao Matao \\
\hline Typhlocybinae & Empoascini & Empoasca sp & -71.980 & -13.510 & 3400 & Cusco \\
\hline Typhlocybinae & Empoascini & Empoasca sp & -71.840 & -13.040 & 3340 & Pampallaqta \\
\hline Typhlocybinae & Empoascini & Empoasca sp & -71.940 & -13.560 & 3100 & Tipón \\
\hline Typhlocybinae & Empoascini & Empoasca sp & -71.959 & -13.522 & 3340 & Perayoc \\
\hline Typhlocybinae & Empoascini & Empoasca sp & -71.870 & -13.540 & 3210 & Kayra \\
\hline Typhlocybinae & Empoascini & Empoasca sp & -72.050 & -13.390 & 3720 & Huatata \\
\hline Typhlocybinae & Empoascini & Empoasca sp & -72.260 & -13.250 & 2842 & Ollantaytambo \\
\hline Typhlocybinae & Empoascini & Empoasca sp & -72.280 & -13.250 & 2800 & Chilca \\
\hline Typhlocybinae & Empoascini & Empoasca sp & -72.080 & -13.090 & 3545 & Cuncani \\
\hline Typhlocybinae & Empoascini & Empoasca sp & -72.070 & -13.110 & 3510 & Tambohuaylla \\
\hline Typhlocybinae & Empoascini & Empoasca sp & -71.230 & -14.250 & 3600 & Marangani \\
\hline Typhlocybinae & Empoascini & Empoasca sp & -71.380 & -14.166 & 3500 & Sicuani \\
\hline Typhlocybinae & Empoascini & Empoasca sp & -72.440 & -13.470 & 2530 & Limatambo \\
\hline Typhlocybinae & Empoascini & Empoasca sp & -72.120 & -13.300 & 2925 & Urubamba \\
\hline Typhlocybinae & Empoascini & Empoasca sp & -72.000 & -13.150 & 3730 & Pampacorral \\
\hline Typhlocybinae & Empoascini & Empoasca sp & -72.140 & -13.460 & 3680 & Izcuchaca \\
\hline Typhlocybinae & Empoascini & Empoasca sp & -71.540 & -13.820 & 3220 & Quiquijana \\
\hline Typhlocybinae & Empoascini & Empoasca sp & -72.040 & -13.010 & 3500 & Ccachin \\
\hline Typhlocybinae & Empoascini & Empoasca sp & -72.020 & -13.050 & 3300 & Choquecancha \\
\hline Typhlocybinae & Empoascini & Empoasca sp & -72.250 & -13.450 & 3300 & Zurite \\
\hline Typhlocybinae & Empoascini & Empoasca sp & -72.250 & -13.480 & 3400 & Yanama \\
\hline Typhlocybinae & Empoascini & Empoasca sp & -71.980 & -13.550 & 3450 & Ccachona \\
\hline Typhlocybinae & Empoascini & Empoasca sp & -71.970 & -13.550 & 3450 & Chocco \\
\hline Typhlocybinae & Empoascini & Empoasca sp & -71.140 & -14.380 & 3600 & Mamuera \\
\hline Typhlocybinae & Empoascini & Empoasca sp & -71.880 & -13.540 & 3240 & San Jerónimo \\
\hline Xestocephalinae & Portanini & Portanus boliviensis* & -71.020 & -14.480 & 4240 & Vilcanota* \\
\hline Xestocephalinae & Portanini & Portanus sp & -71.980 & -13.500 & 3600 & Saqsayhuaman \\
\hline
\end{tabular}

